\title{
[2]Rotaxanes through Palladium Active-Template Oxidative Heck Cross-Couplings
}

James D. Crowley, Kevin Hänni, Ai-Lan Lee, David A. Leigh*

School of Chemistry, University of Edinburgh, The King's Buildings, West Mains Road, Edinburgh EH9 3JJ, UK

* Email: David.Leigh@ed.ac.uk 
1. General Experimental Section $\quad$ S-3

2. Experimental Procedures $\quad$ S-4

3. General Experimental Procedure for the

Oxidative Heck Active-Metal Template Reaction

4. Representative Stacked ${ }^{1}$ H NMR Plots

5. Attempted Active-Template Pd(0)-Catalyzed Heck Cross-Couplings

Table 1 - Round \#1 Optimization Studies: Preliminary Optimization Screens for the Formation of [2]Rotaxane 5

Table 2 - Round \#2 Optimization Studies: Solvent and Oxidant Screen for the Formation of [2]Rotaxane 5

Table 3 - Effect of Lowering Pd(OAc) $)_{2}$ loading on the Formation of [2]Rotaxane 5

6. ${ }^{1}$ H NMR Spectra 


\section{General Experimental Section}

Unless otherwise stated, all reagents were purchased from commercial sources and used without further purification. Dry dichloromethane was obtained by passing the solvent through an activated alumina column on a PureSolv ${ }^{\mathrm{TM}}$ solvent purification system (Innovative Technologies, Inc., MA). Macrocycle 1 was prepared according to literature procedure. ${ }^{1}$ Unless otherwise stated, all reactions were run under an atmosphere of $\mathrm{N}_{2}$. Petrol refers to the fraction of petroleum ether boiling in the range $40-60{ }^{\circ} \mathrm{C}$, IPA refers to isopropyl alcohol. Flash column chromatography was carried out using Kiesegel C60 (Fisher) as the stationary phase, preparative TLC was carried out using precoated silica gel plates (2000 microns thick, Silica gel GF, Uniplate, Germany). Analytical TLC was performed on precoated silica gel plates $(0.25 \mathrm{~mm}$ thick, 60F254, Merck, Germany) and observed under UV light. All ${ }^{1} \mathrm{H}$ and ${ }^{13} \mathrm{C}$ NMR spectra were recorded on a Bruker AV 400 instrument, at a constant temperature of $298 \mathrm{~K}$. Chemical shifts are reported in parts per million and referenced to residual solvent. Coupling constants $(J)$ are reported in hertz $(\mathrm{Hz})$. Standard abbreviations indicating multiplicity were used as follows: $\mathrm{m}$ $=$ multiplet, quint. $=$ quintet, $\mathrm{q}=$ quartet, $\mathrm{t}=$ triplet, $\mathrm{d}=$ doublet, $\mathrm{s}=$ singlet, $\mathrm{br}=$ broad. All melting points were determined using a Sanyo Gallenkamp apparatus and are uncorrected. FAB mass spectrometry was carried out by the mass spectrometry services at the University of Edinburgh. 


\section{Experimental Procedures}

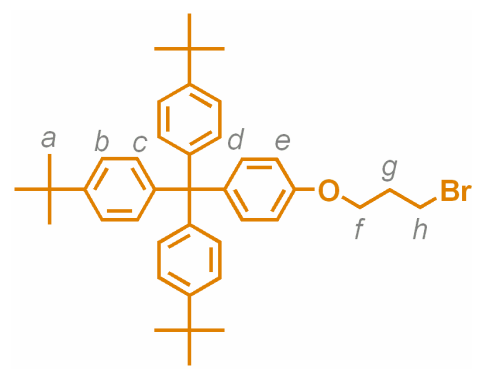

S1

4-[Tris-(4-tert-butylphenyl)methyl]phenol (3.00 g, $5.94 \mathrm{mmol}), 3$-bromo-1-propanol (1.00 mL, $11.9 \mathrm{mmol})$ and triphenylphosphine $(3.20 \mathrm{~g}, 11.9 \mathrm{mmol})$ were dissolved in THF at $0{ }^{\circ} \mathrm{C}$. DIAD (2.30 $\mathrm{mL}, 11.9 \mathrm{mmol})$ was added dropwise. When addition was complete the reaction mixture was allowed to stir for a further $48 \mathrm{~h}$ at RT. The solution was concentrated under reduced pressure, the residue was dissolved in $\mathrm{CH}_{2} \mathrm{Cl}_{2}(10 \mathrm{~mL})$ and precipitated with $\mathrm{MeOH}$. The precipitate was filtered, washed with $\mathrm{MeOH}(3 \times 10 \mathrm{~mL})$ and redissolved in $\mathrm{CH}_{2} \mathrm{Cl}_{2}$. The solution was passed through a plug of silica $\left(\mathrm{CH}_{2} \mathrm{Cl}_{2}\right)$ to afford $\mathbf{S 1}$ as a colorless powder $(2.68 \mathrm{~g}$, 72\%). M.p. $244-246{ }^{\circ} \mathrm{C} .{ }^{1} \mathrm{H}$ NMR $\left(400 \mathrm{MHz}, \mathrm{CDCl}_{3}\right): \delta=7.23\left(\mathrm{~d}, J=8.6 \mathrm{~Hz}, 6 \mathrm{H}, \mathrm{H}_{\mathrm{b}}\right), 7.08(\mathrm{~d}, J$ $=8.6 \mathrm{~Hz}, 8 \mathrm{H}, \mathrm{H}_{\mathrm{c}}$ and $\left.\mathrm{H}_{\mathrm{d}}\right), 6.77\left(\mathrm{~d}, J=8.9 \mathrm{~Hz}, 2 \mathrm{H}, \mathrm{H}_{\mathrm{e}}\right), 4.08\left(\mathrm{t}, J=5.8 \mathrm{~Hz}, 2 \mathrm{H}, \mathrm{H}_{\mathrm{f}}\right), 3.60(\mathrm{t}, J=$ $\left.6.4 \mathrm{~Hz}, 2 \mathrm{H}, \mathrm{H}_{\mathrm{h}}\right), 2.27-2.35\left(\mathrm{~m}, 2 \mathrm{H}, \mathrm{H}_{\mathrm{g}}\right), 1.54\left(\mathrm{~s}, 27 \mathrm{H}, \mathrm{H}_{\mathrm{a}}\right) .{ }^{13} \mathrm{C} \mathrm{NMR}\left(100 \mathrm{MHz}, \mathrm{CDCl}_{3}\right): \delta=$ $156.4,148.3,144.1,139.8,132.2,130.7,124.0,112.9,65.1,63.0,34.3,32.4,31.4,30.2$. LRFAB-MS (3-NOBA matrix): $\mathrm{m} / \mathrm{z}=625.1[\mathrm{M}+\mathrm{H}]^{+}$. 


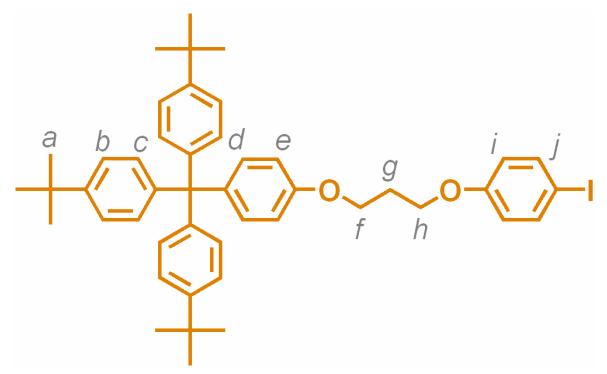

S2

To a solution of $\mathbf{S 1}(1.00 \mathrm{~g}, 1.59 \mathrm{mmol})$ and 4-iodophenol (700 mg, $3.18 \mathrm{mmol})$ in butanone (160 $\mathrm{mL})$ was added potassium carbonate $\left(2.20 \mathrm{~g}, 16.0 \mathrm{mmol}\right.$, . The suspension was heated at $80{ }^{\circ} \mathrm{C}$ for $48 \mathrm{~h}$ under nitrogen atmosphere. After cooling, the suspension was concentrated under reduced pressure and the residue was dissolved in $\mathrm{CH}_{2} \mathrm{Cl}_{2}(50 \mathrm{~mL})$. The organic layer was washed with a solution of $\mathrm{NaOH} 1 \mathrm{M}(3 \times 50 \mathrm{~mL})$ then with water $(1 \times 50 \mathrm{~mL})$, dried $\left(\mathrm{MgSO}_{4}\right)$, filtered and concentrated under reduced pressure. The residue was redissolved in $\mathrm{CH}_{2} \mathrm{Cl}_{2}(10$ $\mathrm{mL}$ ) and precipitated with $\mathrm{MeOH}$. The precipitate was filtered, washed with $\mathrm{MeOH}$ and dried under reduced pressure to yield the aryl iodide $\mathbf{S 2}$ as a colorless solid (1.10 g, 91\%). M. p. 201$203{ }^{\circ} \mathrm{C} .{ }^{1} \mathrm{H}$ NMR $\left(400 \mathrm{MHz}, \mathrm{CDCl}_{3}\right): 7.53\left(\mathrm{~d}, J=8.9 \mathrm{~Hz}, 2 \mathrm{H}, \mathrm{H}_{\mathrm{j}}\right), 7.22\left(\mathrm{~d}, J=8.6 \mathrm{~Hz}, 6 \mathrm{H}, \mathrm{H}_{\mathrm{b}}\right)$, $7.07\left(\mathrm{~d}, J=8.6 \mathrm{~Hz}, 8 \mathrm{H}, \mathrm{H}_{\mathrm{c}}\right.$ and $\left.\mathrm{H}_{\mathrm{d}}\right), 6.76\left(\mathrm{~d}, J=8.9 \mathrm{~Hz}, 2 \mathrm{H}, \mathrm{H}_{\mathrm{i}}\right), 6.68\left(\mathrm{~d}, J=8.9 \mathrm{~Hz}, 2 \mathrm{H}, \mathrm{H}_{\mathrm{e}}\right)$, $4.11\left(\mathrm{t}, J=6.0 \mathrm{~Hz}, 4 \mathrm{H}, \mathrm{H}_{\mathrm{f}}\right.$ and $\left.\mathrm{H}_{\mathrm{h}}\right), 2.19-2.27\left(\mathrm{~m}, 2 \mathrm{H}, \mathrm{H}_{\mathrm{g}}\right), 1.30\left(\mathrm{~s}, 27 \mathrm{H}, \mathrm{H}_{\mathrm{a}}\right) \cdot{ }^{13} \mathrm{C}$ NMR $(100$ $\left.\mathrm{MHz}, \mathrm{CDCl}_{3}\right): \delta=158.7,156.5,148.3,144.1,139.7,138.1,132.2,130.7,124.0,116.9,112.9$, 82.7, 64.6, 64.0, 63.0, 34.3, 31.3, 29.2. LRFAB-MS (3-NOBA matrix): $\mathrm{m} / \mathrm{z}=764[\mathrm{M}+\mathrm{H}]^{+}$; HRFAB-MS (3-NOBA matrix): $\mathrm{m} / \mathrm{z}=764.30903[\mathrm{M}]^{+}$(calcd. for $\mathrm{C}_{46} \mathrm{H}_{53} \mathrm{IO}_{2}, 764.30903$ ). 


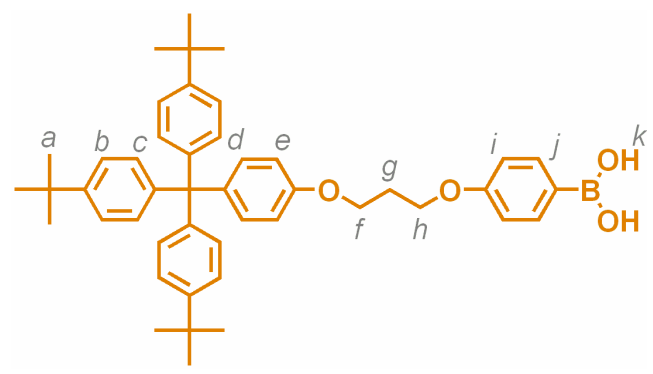

$n$-BuLi, (1.04 mL, 2.5 M solution in hexanes, $2.60 \mathrm{mmol})$ was added dropwise to a solution of S2 $(1.00 \mathrm{~g}, 1.30 \mathrm{mmol})$ in $\mathrm{THF}(20 \mathrm{~mL})$ at $-78{ }^{\circ} \mathrm{C}$. After $30 \mathrm{~min}$ of stirring at $-78^{\circ} \mathrm{C}$, trimethyl borate $(0.73 \mathrm{~mL}, 6.50 \mathrm{mmol})$ was added. The solution was stirred at $-78{ }^{\circ} \mathrm{C}$ for another $2 \mathrm{~h}$ before it was allowed to stir for a further $18 \mathrm{~h}$ at RT. The reaction mixture was quenched with $\mathrm{MeOH}(2$ $\mathrm{mL})$ and the solvents removed under reduced pressure. The residue was dissolved in $\mathrm{CH}_{2} \mathrm{Cl}_{2}(10$ $\mathrm{mL}$ ) and stirred vigorously for $1 \mathrm{~h}$ with $5 \% \mathrm{HCl}(\mathrm{aq}).(10 \mathrm{~mL})$ until the color of the organic layer became orange. The layers were separated and the organic layer washed with water $(50 \mathrm{~mL})$, dried $\left(\mathrm{MgSO}_{4}\right)$, filtered and concentrated under reduced pressure. The residue was purified by column chromatography (gradient $1 \%$ to $5 \%$ of $\mathrm{MeOH}$ in $\mathrm{CH}_{2} \mathrm{Cl}_{2}$ ) to afford the boronic acid 3 as a pale yellow powder $(631 \mathrm{mg}, 71 \%)$. M.p. $184-186{ }^{\circ} \mathrm{C} .{ }^{1} \mathrm{H}$ NMR $\left(400 \mathrm{MHz}, \mathrm{CDCl}_{3} / \mathrm{d}_{6}-\mathrm{DMSO}\right.$ 95:5): $\delta=7.73\left(\mathrm{~d}, J=8.6 \mathrm{~Hz}, 2 \mathrm{H}, \mathrm{H}_{\mathrm{j}}\right), 7.18\left(\mathrm{~d}, J=8.6 \mathrm{~Hz}, 6 \mathrm{H}, \mathrm{H}_{\mathrm{b}}\right), 7.03\left(\mathrm{~d}, J=8.6 \mathrm{~Hz}, 8 \mathrm{H}, \mathrm{H}_{\mathrm{c}}\right.$ and $\left.\mathrm{H}_{\mathrm{d}}\right), 6.85\left(\mathrm{~d}, J=8.6 \mathrm{~Hz}, 2 \mathrm{H}, \mathrm{H}_{\mathrm{i}}\right), 6.72\left(\mathrm{~d}, J=9.0 \mathrm{~Hz}, \mathrm{H}_{\mathrm{e}}\right), 6.14(\mathrm{~s}, 2 \mathrm{H}, \mathrm{OH}), 4.13(\mathrm{t}, J=6.1$ $\left.\mathrm{Hz}, 2 \mathrm{H}, \mathrm{H}_{\mathrm{f}}\right), 4.08\left(\mathrm{t}, J=6.2 \mathrm{~Hz}, 2 \mathrm{H}, \mathrm{H}_{\mathrm{h}}\right), 2.20\left(\mathrm{tt}, J=6.2,6.1 \mathrm{~Hz}, 2 \mathrm{H}, \mathrm{H}_{\mathrm{g}}\right), 1.25\left(\mathrm{~s}, 27 \mathrm{H}, \mathrm{H}_{\mathrm{a}}\right) \cdot{ }^{13} \mathrm{C}$ NMR $\left(100 \mathrm{MHz}, 298 \mathrm{~K}, \mathrm{CDCl}_{3}\right): \delta=156.6,148.2,144.1,139.6,137.4,135.8,132.2,130.6$, $124.0,113.9,113.7,112.9,64.2,63.0,40.1,34.2,31.3,29.3$. 


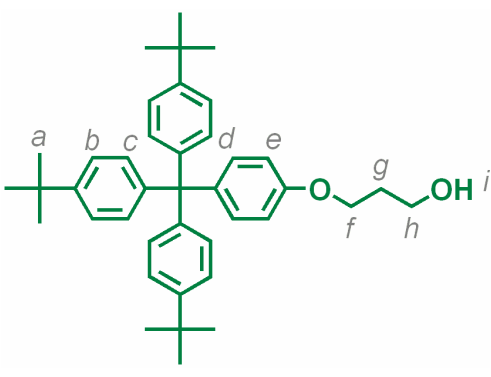

\section{$\mathbf{S 3}^{2}$}

To a solution of 4-[tris-(4-tert-butylphenyl)methyl]phenol ${ }^{3}(2.00 \mathrm{~g}, 4.00 \mathrm{mmol})$ and 1bromopropanol $(0.52 \mathrm{~mL}, 6.00 \mathrm{mmol})$ in butanone $(160 \mathrm{~mL})$ was added potassium carbonate (5.94 g, $39.6 \mathrm{mmol}$.). The suspension was heated at $80{ }^{\circ} \mathrm{C}$ for $48 \mathrm{~h}$ under nitrogen atmosphere. After cooling, the suspension was concentrated under reduced pressure and the residue was dissolved in $\mathrm{CH}_{2} \mathrm{Cl}_{2}(50 \mathrm{~mL})$. The organic layer was washed with water $(3 \times 50 \mathrm{~mL})$, dried $\left(\mathrm{MgSO}_{4}\right)$, filtered and concentrated under reduced pressure. The residue was redissolved in $\mathrm{CH}_{2} \mathrm{Cl}_{2}(10 \mathrm{~mL})$ and precipitated with $\mathrm{MeOH}$. The precipitate was filtered, washed with $\mathrm{MeOH}$ and dried under reduced pressure to yield the alcohol $\mathbf{S 3}$ as a colorless solid (2.05 g, 92\%). M.p. 290-292 ${ }^{\circ} \mathrm{C} .{ }^{1} \mathrm{H}$ NMR $\left(400 \mathrm{MHz}, \mathrm{CDCl}_{3}\right): \delta=7.23\left(\mathrm{~d}, J=8.6 \mathrm{~Hz}, 6 \mathrm{H}, \mathrm{H}_{\mathrm{b}}\right), 7.08(\mathrm{~d}, J=8.6 \mathrm{~Hz}$, $8 \mathrm{H}, \mathrm{H}_{\mathrm{c}}$ and $\left.\mathrm{H}_{\mathrm{d}}\right), 6.77\left(\mathrm{~d}, J=8.9 \mathrm{~Hz}, 2 \mathrm{H}, \mathrm{H}_{\mathrm{e}}\right), 4.10\left(\mathrm{t}, J=5.9 \mathrm{~Hz}, 2 \mathrm{H}, \mathrm{H}_{\mathrm{f}}\right), 3.86(\mathrm{q}, J=4.8 \mathrm{~Hz}, 2 \mathrm{H}$, $\left.\mathrm{H}_{\mathrm{h}}\right), 2.04\left(\mathrm{~m}, 2 \mathrm{H}, \mathrm{H}_{\mathrm{g}}\right), 1.78\left(\mathrm{t}, J=4.8 \mathrm{~Hz}, 1 \mathrm{H}, \mathrm{H}_{\mathrm{i}}\right), 1.30\left(\mathrm{~s}, 27 \mathrm{H}, \mathrm{H}_{\mathrm{a}}\right) \cdot{ }^{13} \mathrm{C}$ NMR (100 MHz, $\left.\mathrm{CDCl}_{3}\right): \delta=156.4,148.2,144.0,139.8,132.2,130.6,124.0,112.9,65.7,63.0,60.7,34.2,31.9$, 31.3. LRFAB-MS (3-NOBA matrix): $m / z=562.4[\mathrm{M}]^{+}$. 


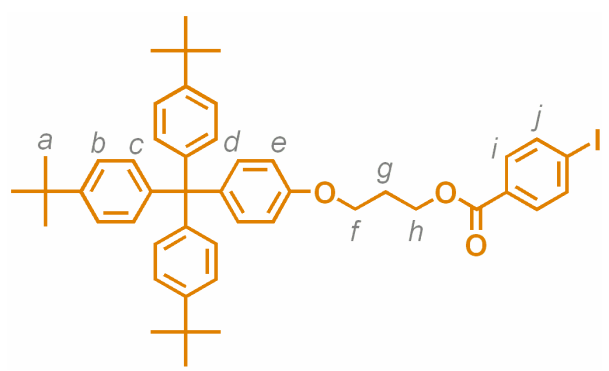

S4

4-Iodobenzoic acid (264 mg, $1.07 \mathrm{mmol})$, DMAP $(6.50 \mathrm{mg}, 53.0 \mu \mathrm{mol})$ and EDC (118 mg, 0.61 mmol) were added successively to a solution of the alcohol $\mathbf{S 3}(300 \mathrm{mg}, 0.53 \mathrm{mmol})$ in $\mathrm{CH}_{2} \mathrm{Cl}_{2}$ $(10 \mathrm{~mL})$. The resulting solution was allowed to stir at RT for $19 \mathrm{~h}$. The reaction mixture was quenched by addition of water, the aqueous layer extracted with $\mathrm{CH}_{2} \mathrm{Cl}_{2}(\mathrm{x} 3)$ and the combined organic layers dried $\left(\mathrm{MgSO}_{4}\right)$. Purification by flash column chromatography (10:1 hexane:ethyl acetate) yielded the title compound $\mathbf{S 4}$ as a colorless solid (300 mg, 71\%). Mp $232-234{ }^{\circ} \mathrm{C} .{ }^{1} \mathrm{H}$ $\operatorname{NMR}\left(400 \mathrm{MHz}, \mathrm{CDCl}_{3}\right): \delta=7.79\left(\mathrm{~d}, J=8.6 \mathrm{~Hz}, 2 \mathrm{H}, \mathrm{H}_{\mathrm{i}}\right), 7.73\left(\mathrm{~d}, J=8.6 \mathrm{~Hz}, 2 \mathrm{H}, \mathrm{H}_{\mathrm{j}}\right), 7.23(\mathrm{~d}, J$ $\left.=8.6 \mathrm{~Hz}, 6 \mathrm{H}, \mathrm{H}_{\mathrm{b}}\right), 7.08\left(\mathrm{~d}, J=9.0 \mathrm{~Hz}, 2 \mathrm{H}, \mathrm{H}_{\mathrm{d}}\right), 7.07\left(\mathrm{~d}, J=8.6 \mathrm{~Hz}, 6 \mathrm{H}, \mathrm{H}_{\mathrm{c}}\right), 6.76(\mathrm{~d}, J=9.0 \mathrm{~Hz}$, $\left.2 \mathrm{H}, \mathrm{H}_{\mathrm{e}}\right), 4.51\left(\mathrm{t}, J=6.3 \mathrm{~Hz}, 2 \mathrm{H}, \mathrm{H}_{\mathrm{h}}\right), 4.10\left(\mathrm{t}, J=6.1 \mathrm{~Hz}, 2 \mathrm{H}, \mathrm{H}_{\mathrm{f}}\right), 2.27-2.20\left(\mathrm{~m}, 2 \mathrm{H}, \mathrm{H}_{\mathrm{g}}\right), 1.30(\mathrm{~s}$, $\left.27 \mathrm{H}, \mathrm{H}_{\mathrm{a}}\right) .{ }^{13} \mathrm{C} \mathrm{NMR}\left(100 \mathrm{MHz}, 298 \mathrm{~K}, \mathrm{CDCl}_{3}\right): \delta=166.0,156.5,148.3,144.1,139.8,137.7$, 132.3, 131.0, 130.7, 129.7, 124.0, 112.9, 100.7, 64.1, 63.0, 62.2, 34.3, 31.4, 28.7. LRFAB-MS (3-NOBA matrix): $m / z=792[\mathrm{M}]^{+}$; HRFAB-MS (3-NOVA matrix): $m / z=792.3024$ (calc. for $\left.\mathrm{C}_{47} \mathrm{H}_{53} \mathrm{O}_{3} \mathrm{I} 792.3040\right)$. 


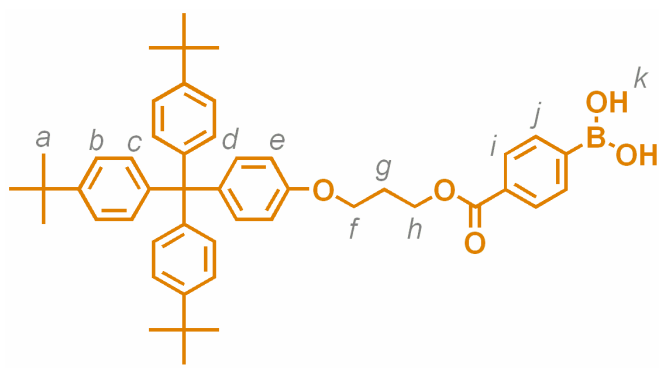

11

To a solution of $\mathbf{S 4}(100 \mathrm{mg}, 0.12 \mathrm{mmol})$ and bis-(2-dimethylaminoethyl)-ether (0.028 $\mathrm{mL}, 0.15$ $\mathrm{mmol})$ in THF $(1 \mathrm{~mL})$ was added isopropylmagnesium chloride $(0.075 \mathrm{~mL}, 0.15 \mathrm{mmol})$ at $15^{\circ} \mathrm{C}$. The mixture was stirred at RT for $20 \mathrm{~min}$ and trimethylborate $(0.067 \mathrm{~mL}, 0.6 \mathrm{mmol})$ was added at $0{ }^{\circ} \mathrm{C}$. The mixture was then quenched with $\mathrm{HCl} 1 \mathrm{M}(2 \mathrm{~mL})$, extracted with $\mathrm{CH}_{2} \mathrm{Cl}_{2}(3 \mathrm{~mL})$ and the organic phase was dried $\left(\mathrm{MgSO}_{4}\right)$, filtered and concentrated under reduced pressure. The residue was purified by column chromatography $\left(\mathrm{CH}_{2} \mathrm{Cl}_{2} / \mathrm{MeOH} 100: 0\right.$ to $\left.95: 5\right)$ to afford the boronic acid 11 as a colorless powder (66 mg, 77\%). M.p 192-194 ${ }^{\circ} \mathrm{C} .{ }^{1} \mathrm{H}$ NMR $(400 \mathrm{MHz}$, $\mathrm{CDCl}_{3} / \mathrm{d}_{6}$-DMSO 97:3): $\delta=7.91\left(\mathrm{~d}, J=8.0 \mathrm{~Hz}, 2 \mathrm{H}, \mathrm{H}_{\mathrm{i}}\right), 7.83\left(\mathrm{~d}, J=8.1 \mathrm{~Hz}, 2 \mathrm{H}, \mathrm{H}_{\mathrm{j}}\right), 7.13(\mathrm{~d}, J=$ $\left.8.5 \mathrm{~Hz}, 6 \mathrm{H}, \mathrm{H}_{\mathrm{b}}\right), 6.98\left(\mathrm{~d}, J=8.4 \mathrm{~Hz}, 8 \mathrm{H}, \mathrm{H}_{\mathrm{c}}\right.$ and $\left.\mathrm{H}_{\mathrm{d}}\right), 6.68\left(\mathrm{~d}, J=8.8 \mathrm{~Hz}, 2 \mathrm{H}, \mathrm{H}_{\mathrm{e}}\right), 6.36(\mathrm{~s}, 2 \mathrm{H}$, $\left.\mathrm{H}_{\mathrm{k}}\right), 4.41\left(\mathrm{t}, J=6.1 \mathrm{~Hz}, 2 \mathrm{H}, \mathrm{H}_{\mathrm{h}}\right), 4.02\left(\mathrm{t}, J=6.0 \mathrm{~Hz}, 2 \mathrm{H}, \mathrm{H}_{\mathrm{f}}\right), 2.10-2.20\left(\mathrm{~m}, 2 \mathrm{H}, \mathrm{H}_{\mathrm{g}}\right), 1.20(\mathrm{~s}$, 27H, $\left.\mathrm{H}_{\mathrm{a}}\right) .{ }^{13} \mathrm{C} \mathrm{NMR}\left(100 \mathrm{MHz}, \mathrm{CDCl}_{3} / \mathrm{d}_{6}\right.$-DMSO 9:1): 156.2, 154.2, 148.0, 143.8, 139.4, 133.9, $131.9,130.4,128.2,123.8,112.7,111.0,106.9,95.3,63.9,61.6,34.0,31.1,28.5$. 


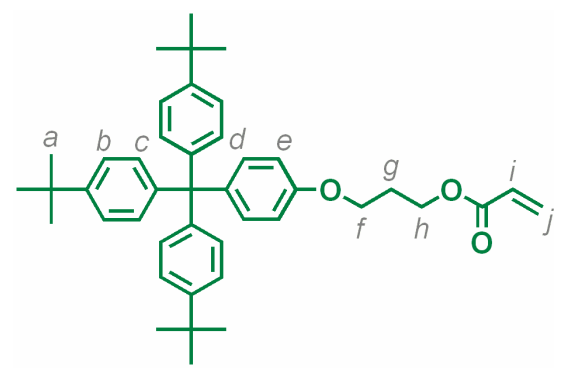

4

Triethylamine (1.86 mL, $13.3 \mathrm{mmol})$ was added to a solution of alcohol $\mathbf{S 3}(1.50 \mathrm{~g}, 2.67 \mathrm{mmol})$ in $\mathrm{CH}_{2} \mathrm{Cl}_{2}(40 \mathrm{~mL})$ and the resulting mixture was cooled to $0{ }^{\circ} \mathrm{C}$. Acryloyl chloride $(0.54 \mathrm{~mL}$, $6.66 \mathrm{mmol}$ ) and DMAP (49 $\mathrm{mg}, 0.40 \mathrm{mmol}$ ) was added. The resulting mixture was allowed to stir at $0{ }^{\circ} \mathrm{C}$ for $2 \mathrm{~h}$ before the reaction was quenched by addition of water. The aqueous layer was extracted with $\mathrm{CH}_{2} \mathrm{Cl}_{2}$ (x3), washed with brine and dried $\left(\mathrm{MgSO}_{4}\right)$. Purification by column chromatography (3:1 hexane:ethyl acetate) provided the alkene $\mathbf{4}$ as a colorless solid (1.04 g, 63\%). M.p. $210-212{ }^{\circ} \mathrm{C} .{ }^{1} \mathrm{H}$ NMR (400 MHz, $\left.\mathrm{CDCl}_{3}\right): \delta 7.23\left(\mathrm{~d}, J=8.6 \mathrm{~Hz}, 6 \mathrm{H}, \mathrm{H}_{\mathrm{b}}\right), 7.08(\mathrm{~d}, J=$ $8.6 \mathrm{~Hz}, 8 \mathrm{H}, \mathrm{H}_{\mathrm{c}}$ and $\left.\mathrm{H}_{\mathrm{d}}\right), 7.76\left(\mathrm{~d}, J=8.9 \mathrm{~Hz}, 2 \mathrm{H}, \mathrm{H}_{\mathrm{e}}\right), 6.40\left(\mathrm{~d}, J=17.3 \mathrm{~Hz}, 1 \mathrm{H}, \mathrm{H}_{\mathrm{j}}\right.$-trans $), 6.12(\mathrm{dd}$, $J=17.3$ and $\left.11.9 \mathrm{~Hz}, 1 \mathrm{H}, \mathrm{H}_{\mathrm{i}}\right), 5.82\left(\mathrm{~d}, J=11.9 \mathrm{~Hz}, 1 \mathrm{H}, \mathrm{H}_{\mathrm{j}}-c i s\right), 4.36\left(\mathrm{t}, J=6.3 \mathrm{~Hz}, 2 \mathrm{H}, \mathrm{H}_{\mathrm{h}}\right)$, $4.04\left(\mathrm{t}, J=6.1 \mathrm{~Hz}, 2 \mathrm{H}, \mathrm{H}_{\mathrm{f}}\right), 2.19-2.10\left(\mathrm{~m}, 2 \mathrm{H}, \mathrm{H}_{\mathrm{g}}\right), 1.30\left(\mathrm{~s}, 27 \mathrm{H}, \mathrm{H}_{\mathrm{a}}\right) .{ }^{13} \mathrm{C}$ NMR $(100 \mathrm{MHz}, 298$ $\left.\mathrm{K}, \mathrm{CDCl}_{3}\right): \delta=166.2,156.6,148.3,144.1,139.8,139.5,132.3,130.7,128.4,124.0,113.0,64.1$, 63.1, 61.5, 34.3, 31.4, 28.7. LRFAB-MS (3-NOBA matrix): $m / z=616[\mathrm{M}]^{+}$; HRFAB-MS (3NOBA matrix): $m / z=616.3928$ (calc. for $\mathrm{C}_{43} \mathrm{H}_{52} \mathrm{O}_{3} 616.3917$ ). 


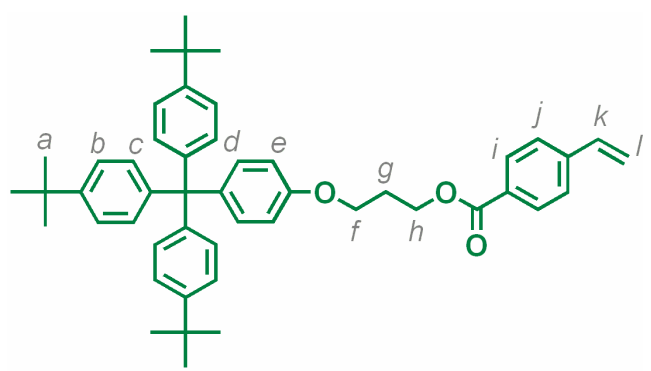

8

A solution of alcohol $\mathbf{S 3}(1.00 \mathrm{~g}, 1.72 \mathrm{mmol})$ and 4-vinylbenzoic acid (310 mg, $2.06 \mathrm{mmol})$ in $\mathrm{CH}_{2} \mathrm{Cl}_{2}(50 \mathrm{~mL})$ was cooled to $0{ }^{\circ} \mathrm{C}$. EDCI $(430 \mathrm{mg}, 2.25 \mathrm{mmol})$ and DMAP $(210 \mathrm{mg}, 1.72$ mmol) were added. The resulting mixture was allowed to stir overnight at RT. The reaction was quenched by addition of water and the resulting aqueous layer extracted with $\mathrm{CH}_{2} \mathrm{Cl}_{2}(\mathrm{x} 3)$. The combined organic layers were dried over anhydrous $\mathrm{MgSO}_{4}$. Purification by column chromatography (9:1 hexane:ethyl acetate) provided the alkene $\mathbf{8}$ as a colorless solid $(850 \mathrm{mg}$, 72\%). M.p. $180-183{ }^{\circ} \mathrm{C} .{ }^{1} \mathrm{H}$ NMR $\left(400 \mathrm{MHz}, \mathrm{CDCl}_{3}\right): \delta=7.91\left(\mathrm{~d}, J=8.3 \mathrm{~Hz}, 2 \mathrm{H}, \mathrm{H}_{\mathrm{i}}\right), 7.36(\mathrm{~d}, J$ $\left.=8.3 \mathrm{~Hz}, 2 \mathrm{H}, \mathrm{H}_{\mathrm{j}}\right), 7.14\left(\mathrm{~d}, J=8.5 \mathrm{~Hz}, 6 \mathrm{H}, \mathrm{H}_{\mathrm{b}}\right), 7.00\left(\mathrm{~d}, J=8.5 \mathrm{~Hz}, 6 \mathrm{H}, \mathrm{H}_{\mathrm{c}}\right), 7.00(\mathrm{~d}, J=8.8 \mathrm{~Hz}$, $\left.2 \mathrm{H}, \mathrm{H}_{\mathrm{d}}\right), 6.69\left(\mathrm{~d}, J=8.8 \mathrm{~Hz}, 2 \mathrm{H}, \mathrm{H}_{\mathrm{e}}\right), 6.66\left(\mathrm{dd}, J=17.6,10.9 \mathrm{~Hz}, 1 \mathrm{H}, \mathrm{H}_{\mathrm{k}}\right), 5.77(\mathrm{~d}, J=17.6 \mathrm{~Hz}$, 1H, $\mathrm{H}_{\mathrm{l}}$-trans), $5.29\left(\mathrm{~d}, J=10.9 \mathrm{~Hz}, 1 \mathrm{H}, \mathrm{H}_{\mathrm{l}}\right.$-cis $), 4.43\left(\mathrm{t}, J=6.2 \mathrm{~Hz}, 2 \mathrm{H}, \mathrm{H}_{\mathrm{h}}\right), 4.02(\mathrm{t}, J=6.1 \mathrm{~Hz}$, $\left.2 \mathrm{H}, \mathrm{H}_{\mathrm{f}}\right), 2.16\left(\mathrm{tt}, J=6.2,6.1 \mathrm{~Hz}, 2 \mathrm{H}, \mathrm{H}_{\mathrm{g}}\right), 1.21\left(\mathrm{~s}, 27 \mathrm{H}, \mathrm{H}_{\mathrm{a}}\right) .{ }^{13} \mathrm{C} \mathrm{NMR}(100 \mathrm{MHz}, 298 \mathrm{~K}$, $\left.\mathrm{CDCl}_{3}\right): \delta=166.3,156.6,148.3,144.1,142.0,139.8,136.0,132.3,130.7,129.9,129.4,126.1$, 124.3, 116.5, 113.0, 64.3, 63.1, 61.9, 34.3, 31.4, 28.9. LRFAB-MS (3-NOBA matrix): $m / z=$ $692.8[\mathrm{M}]^{+}$; HRFAB-MS (3-NOBA matrix): $\mathrm{m} / z=692.4226[\mathrm{M}]^{+}$(calc. for $\mathrm{C}_{49} \mathrm{H}_{56} \mathrm{O}_{3} 692.4230$ ). 


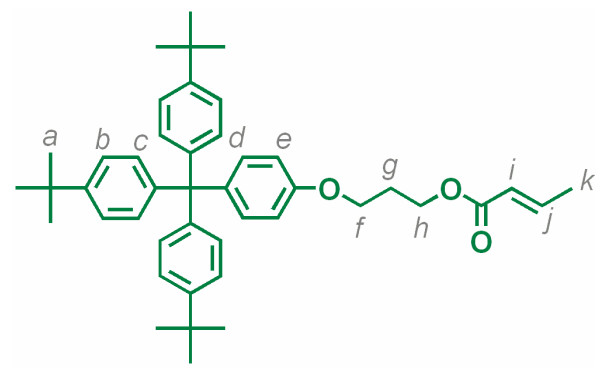

13

A solution of alcohol $\mathbf{S 3}(1.00 \mathrm{~g}, 1.78 \mathrm{mmol})$ and triethylamine $(1.24 \mathrm{~mL}, 8.88 \mathrm{mmol})$ in $\mathrm{CH}_{2} \mathrm{Cl}_{2}$ $(30 \mathrm{~mL})$ was cooled to $0{ }^{\circ} \mathrm{C}$. Crotonyl chloride $(0.43 \mathrm{~mL}, 4.44 \mathrm{mmol})$ and DMAP $(33 \mathrm{mg}, 0.267$ mmol) was added before the reaction mixture was allowed to stir overnight at RT. The reaction was quenched by addition of water and the resulting aqueous layer extracted with $\mathrm{CH}_{2} \mathrm{Cl}_{2}$ (x3). The combined organic layers was with brine and dried $\left(\mathrm{MgSO}_{4}\right)$. Purification by column chromatography (10:1 hexane:ethyl acetate) provided the alkene $\mathbf{S 5}$ as a pale yellow solid (593 mg, 53\%). ${ }^{1} \mathrm{H}$ NMR (400 MHz, $\left.\mathrm{CDCl}_{3}\right): \delta=7.23\left(\mathrm{~d}, J=8.7 \mathrm{~Hz}, 6 \mathrm{H}, \mathrm{H}_{\mathrm{b}}\right), 7.08(\mathrm{~d}, J=8.7 \mathrm{~Hz}, 6 \mathrm{H}$, $\left.\mathrm{H}_{\mathrm{c}}\right), 7.08\left(\mathrm{~d}, J=8.9 \mathrm{~Hz}, 2 \mathrm{H}, \mathrm{H}_{\mathrm{d}}\right), 6.75(\mathrm{~d}, J=8.9 \mathrm{~Hz}, 2 \mathrm{H}$, $\left.\mathrm{H}_{\mathrm{e}}\right), 5.92\left(\mathrm{~m}, 1 \mathrm{H}, \mathrm{H}_{\mathrm{j}}\right), 5.16\left(\mathrm{~m}, 2 \mathrm{H}, \mathrm{H}_{\mathrm{k}}\right), 4.29(\mathrm{t}, J=6.3$ $\left.\mathrm{Hz}, 2 \mathrm{H}, \mathrm{H}_{\mathrm{h}}\right), 4.02\left(\mathrm{t}, J=6.1 \mathrm{~Hz}, 2 \mathrm{H}, \mathrm{H}_{\mathrm{f}}\right), 3.10(\mathrm{dt}, J=$ 7.0, $\left.1.4 \mathrm{~Hz}, 2 \mathrm{H}, \mathrm{H}_{\mathrm{i}}\right), 2.11\left(\mathrm{tt}, J=6.3,6.1 \mathrm{~Hz}, 2 \mathrm{H}, \mathrm{H}_{\mathrm{g}}\right)$, $1.30\left(\mathrm{~s}, 27 \mathrm{H}, \mathrm{H}_{\mathrm{a}}\right)$.

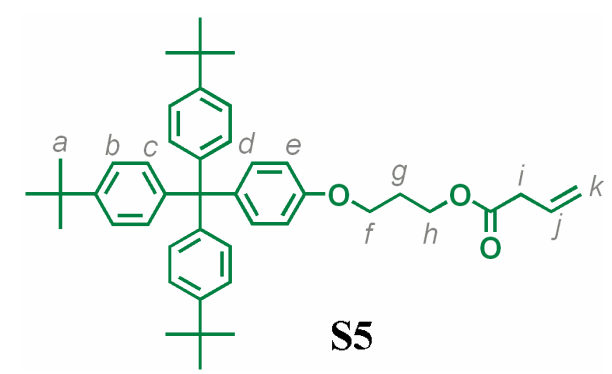

DBU $(0.18 \mathrm{~mL}, 1.19 \mathrm{mmol})$ was added dropwise to a solution of $\mathbf{S 5}(593 \mathrm{mg}, 0.940 \mathrm{mmol})$ in THF (12 mL) at $0{ }^{\circ} \mathrm{C}$. The resulting mixture was allowed to warm slowly to $\mathrm{RT}$ and stirred overnight. THF was removed under reduced pressure and the resulting oil redissolved in $\mathrm{CH}_{2} \mathrm{Cl}_{2}$. The organic layer was washed with $1 \mathrm{M} \mathrm{HCl}$ (aq.) (x3), the aqueous layer extracted with $\mathrm{CH}_{2} \mathrm{Cl}_{2}$ 
(x3) and the combined organic layers washed with dilute $\mathrm{NaOH}$ (aq.), $1 \mathrm{M} \mathrm{HCl}$ (aq.), water and dried $\left(\mathrm{MgSO}_{4}\right)$ to yield the alkene 13 as a pale yellow solid. M.p. $185-188{ }^{\circ} \mathrm{C} .{ }^{1} \mathrm{H}$ NMR $(400$ $\left.\mathrm{MHz}, \mathrm{CDCl}_{3}\right): \delta=7.13\left(\mathrm{~d}, J=8.5 \mathrm{~Hz}, 6 \mathrm{H}, \mathrm{H}_{\mathrm{b}}\right), 6.99\left(\mathrm{~d}, J=8.5 \mathrm{~Hz}, 6 \mathrm{H}, \mathrm{H}_{\mathrm{c}}\right), 6.99(\mathrm{~d}, J=8.9 \mathrm{~Hz}$, $\left.2 \mathrm{H}, \mathrm{H}_{\mathrm{d}}\right), 6.88\left(\mathrm{dq}, J=15.5,6.9 \mathrm{~Hz}, 1 \mathrm{H}, \mathrm{H}_{\mathrm{j}}\right), 6.66\left(\mathrm{~d}, J=8.9 \mathrm{~Hz}, 2 \mathrm{H}, \mathrm{H}_{\mathrm{e}}\right), 5.75(\mathrm{dq}, J=15.5,1.6$ $\left.\mathrm{Hz}, 1 \mathrm{H}, \mathrm{H}_{\mathrm{i}}\right), 4.22\left(\mathrm{t}, J=6.2 \mathrm{~Hz}, 2 \mathrm{H}, \mathrm{H}_{\mathrm{h}}\right), 3.93\left(\mathrm{t}, J=6.1 \mathrm{~Hz}, 2 \mathrm{H}, \mathrm{H}_{\mathrm{f}}\right), 2.03(\mathrm{tt}, J=6.2,6.1 \mathrm{~Hz}, 2 \mathrm{H}$, $\left.\mathrm{H}_{\mathrm{g}}\right), 1.77\left(\mathrm{dd}, J=6.9,1.6 \mathrm{~Hz}, 2 \mathrm{H}, \mathrm{H}_{\mathrm{k}}\right), 1.21\left(\mathrm{~s}, 27 \mathrm{H}, \mathrm{H}_{\mathrm{a}}\right) \cdot{ }^{13} \mathrm{C} \mathrm{NMR}\left(100 \mathrm{MHz}, 298 \mathrm{~K}, \mathrm{CDCl}_{3}\right): \delta$ $=166.5,156.6,148.3,144.8,144.2,139.7,132.3,130.4,123.7,122.6,113.0,64.2,63.1,61.1$, 34.3, 31.4, 28.8, 18.0. LRFAB-MS (3-NOBA matrix): $m / z=630.8[\mathrm{M}]^{+}$; HRFAB-MS (3-NOBA matrix): $m / z=630.4072[\mathrm{M}]^{+}$(calc. for $\mathrm{C}_{44} \mathrm{H}_{54} \mathrm{O}_{3} 630.4073$ ). 


\section{General Experimental Procedure for the Oxidative Heck Active-Metal Template Reaction:}

A solution of the alkene half-thread (1 equiv.), boronic acid half-thread (2 equiv.), bipy macrocycle 1 ( 1 equiv.), $\mathrm{Pd}(\mathrm{OAc})_{2}(0.1$ equiv.) and benzoquinone (1 equiv.) in a 1:1 mixture of $\mathrm{CHCl}_{3} / \mathrm{CH}_{2} \mathrm{Cl}_{2}$ was allowed to stir under a balloon of $\mathrm{O}_{2}$. After $72 \mathrm{~h}$, a solution of $\mathrm{KCN}$ (10 equiv.) in $\mathrm{MeOH}$ was added and the resulting suspension allowed to stir at RT for $1 \mathrm{~h}$. The solvents were removed under reduced pressure and the resulting crude mixture dissolved in $\mathrm{CH}_{2} \mathrm{Cl}_{2}$ and water. The aqueous layer was extracted with $\mathrm{CH}_{2} \mathrm{Cl}_{2}$ (x3), washed with brine and dried $\left(\mathrm{MgSO}_{4}\right)$. Purification by column chromatography (5:4.5:0.5 hexane: $\left.\mathrm{CH}_{2} \mathrm{Cl}_{2}: \mathrm{MeCN}\right)$ provided the rotaxane as a colorless film.

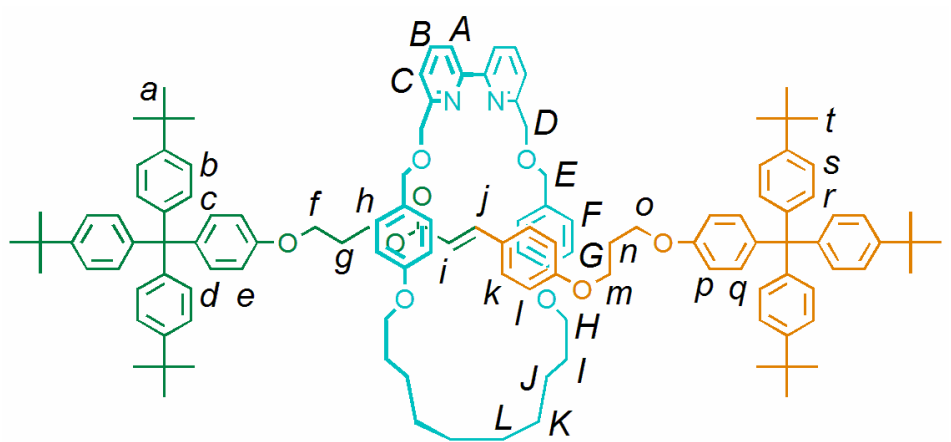

5

${ }^{1} \mathrm{H}$ NMR $\left(400 \mathrm{MHz}, \mathrm{CDCl}_{3}\right): \delta=7.96\left(\mathrm{~d}, J=7.6 \mathrm{~Hz}, 2 \mathrm{H}, \mathrm{H}_{\mathrm{A}}\right), 7.49(\mathrm{dd}, J=7.8,7.6 \mathrm{~Hz}, 2 \mathrm{H}$, $\left.\mathrm{H}_{\mathrm{B}}\right), 7.39\left(\mathrm{~d}, J=15.9 \mathrm{~Hz}, 1 \mathrm{H}, \mathrm{H}_{\mathrm{j}}\right), 7.28(\mathrm{~d}, J=7.8 \mathrm{~Hz}, 2 \mathrm{H}, \mathrm{Hc}), 7.23\left(\mathrm{~d}, J=8.7 \mathrm{~Hz}, 12 \mathrm{H}, \mathrm{H}_{\mathrm{b}}, \mathrm{H}_{\mathrm{s}}\right)$, 7.10-6.99 (m, 22H, $\left.\mathrm{H}_{\mathrm{c}}, \mathrm{H}_{\mathrm{r}}, \mathrm{H}_{\mathrm{F}}, \mathrm{H}_{\mathrm{d}}, \mathrm{H}_{\mathrm{q}}, \mathrm{H}_{\mathrm{k}}\right), 6.62\left(\mathrm{~d}, J=8.6 \mathrm{~Hz}, 6 \mathrm{H}, \mathrm{H}_{\mathrm{G}}, \mathrm{H}_{\mathrm{e}}\right), 6.56(\mathrm{~d}, J=8.9 \mathrm{~Hz}$, 2H, $\left.\mathrm{H}_{\mathrm{p}}\right), 6.39\left(\mathrm{~d}, J=8.7 \mathrm{~Hz}, 2 \mathrm{H}, \mathrm{H}_{\mathrm{l}}\right), 6.04\left(\mathrm{~d}, J=15.9 \mathrm{~Hz}, 1 \mathrm{H}, \mathrm{H}_{\mathrm{i}}\right), 4.56\left(\mathrm{~s}, 4 \mathrm{H}, \mathrm{H}_{\mathrm{D} / \mathrm{E}}\right), 4.55(\mathrm{~s}$, $\left.4 \mathrm{H}, \mathrm{H}_{\mathrm{D} / \mathrm{E}}\right), 4.24\left(\mathrm{t}, J=6.2 \mathrm{~Hz}, 2 \mathrm{H}, \mathrm{H}_{\mathrm{h}}\right), 3.85\left(\mathrm{t}, J=6.2 \mathrm{~Hz}, 2 \mathrm{H}, \mathrm{H}_{\mathrm{f}}\right), 3.74\left(\mathrm{t}, J=6.2 \mathrm{~Hz}, 6 \mathrm{H}, \mathrm{H}_{\mathrm{H}}\right.$, $\left.\mathrm{H}_{\mathrm{m} / \mathrm{o}}\right), 3.64\left(\mathrm{t}, J=6.2 \mathrm{~Hz}, 2 \mathrm{H}, \mathrm{H}_{\mathrm{m} / \mathrm{o}}\right), 2.00\left(\mathrm{tt}, J=6.2,6.2 \mathrm{~Hz}, 2 \mathrm{H}, \mathrm{H}_{\mathrm{g}}\right), 1.85(\mathrm{tt}, J=6.2,6.2 \mathrm{~Hz}$ 
$\left.2 \mathrm{H}, \mathrm{H}_{\mathrm{n}}\right), 1.59-1.53\left(\mathrm{~m}, 4 \mathrm{H}, \mathrm{H}_{\mathrm{I}}\right), 1.30\left(\mathrm{~s}, 54 \mathrm{H}, \mathrm{H}_{\mathrm{a}}, \mathrm{H}_{\mathrm{t}}\right), 1.26-1.05\left(\mathrm{~m}, 12 \mathrm{H}, \mathrm{H}_{\mathrm{J}}, \mathrm{H}_{\mathrm{K}}, \mathrm{H}_{\mathrm{L}}\right) .{ }^{13} \mathrm{C}$ NMR $\left(100 \mathrm{MHz}, 298 \mathrm{~K}, \mathrm{CDCl}_{3}\right): \delta=167.1,160.3,158.5,158.2,156.5,156.4,155.1,148.2,148.2$, $144.5,144.1(\times 2), 139.5,139.4(\times 2), 136.8,132.1,132.0,130.6,129.7,129.3,126.6,124.0$, 124.0, 121.0, 119.5, 114.9, 114.3, 114.2, $112.8(\times 2), 112.8,72.4,72.0,67.5,64.2,63.9,63.8$, $63.0(\times 2), 61.0,34.2(\times 2), 31.3(\times 2), 29.7,29.3,28.9,28.7(\times 2), 25.7$; LRFAB-MS (3-NOBA matrix): $m / z=1821[\mathrm{M}+\mathrm{H}]^{+}$; HRFAB-MS (3-NOBA matrix): $m / z=1820.1117[\mathrm{M}+\mathrm{H}]^{+}($calc. for $\mathrm{C}_{125} \mathrm{H}_{147} \mathrm{~N}_{2} \mathrm{O}_{9}$ 1820.1107).

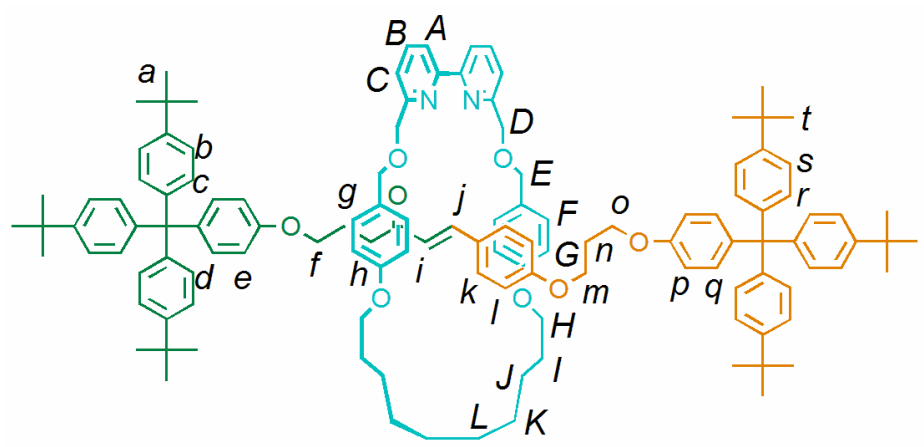

9

${ }^{1} \mathrm{H}$ NMR (400 MHz, $\left.\mathrm{CDCl}_{3}\right): \delta=7.94\left(\mathrm{~d}, J=7.7 \mathrm{~Hz}, 2 \mathrm{H}, \mathrm{H}_{\mathrm{A}}\right), 7.48(\mathrm{dd}, J=7.7,7.7 \mathrm{~Hz}, 2 \mathrm{H}$, $\left.\mathrm{H}_{\mathrm{B}}\right), 7.27(\mathrm{~d}, J=7.7 \mathrm{~Hz}, 2 \mathrm{H}, \mathrm{Hc}), 7.23\left(\mathrm{~d}, J=8.5 \mathrm{~Hz}, 12 \mathrm{H}, \mathrm{H}_{\mathrm{b}}, \mathrm{H}_{\mathrm{s}}\right), 7.18\left(\mathrm{~d}, J=16.3 \mathrm{~Hz}, 1 \mathrm{H}, \mathrm{H}_{\mathrm{j}}\right)$, 7.10-6.98 (m, 20H, $\left.\mathrm{H}_{\mathfrak{c}}, \mathrm{H}_{\mathrm{r}}, \mathrm{H}_{\mathrm{F}}, \mathrm{H}_{\mathrm{d}}, \mathrm{H}_{\mathrm{q}}\right), 6.99\left(\mathrm{~d}, J=8.7 \mathrm{~Hz}, 2 \mathrm{H}, \mathrm{H}_{\mathrm{k}}\right), 6.65-6.57\left(\mathrm{~m}, 8 \mathrm{H}, \mathrm{H}_{\mathrm{G}}, \mathrm{H}_{\mathrm{e}}\right.$, $\left.\mathrm{H}_{\mathrm{p}}\right), 6.41\left(\mathrm{~d}, J=8.7 \mathrm{~Hz}, 2 \mathrm{H}, \mathrm{H}_{1}\right), 6.27\left(\mathrm{~d}, J=16.3 \mathrm{~Hz}, 1 \mathrm{H}, \mathrm{H}_{\mathrm{i}}\right), 4.55\left(\mathrm{~s}, 8 \mathrm{H}, \mathrm{H}_{\mathrm{D}}, \mathrm{H}_{\mathrm{E}}\right), 3.80(\mathrm{t}, J=$ $\left.6.2 \mathrm{~Hz}, 2 \mathrm{H}, \mathrm{H}_{\mathrm{f}}\right), 3.78\left(\mathrm{t}, J=6.2 \mathrm{~Hz}, 2 \mathrm{H}, \mathrm{H}_{\mathrm{m} / \mathrm{o}}\right), 3.74\left(\mathrm{t}, J=6.4 \mathrm{~Hz}, 4 \mathrm{H}, \mathrm{H}_{\mathrm{H}}\right), 3.69(\mathrm{t}, J=6.2 \mathrm{~Hz}$, $\left.2 \mathrm{H}, \mathrm{H}_{\mathrm{m} / \mathrm{o}}\right), 2.58\left(\mathrm{t}, J=7.1,2 \mathrm{H}, \mathrm{H}_{\mathrm{h}}\right), 1.95\left(\mathrm{tt}, J=7.1,6.2 \mathrm{~Hz}, 2 \mathrm{H}, \mathrm{H}_{\mathrm{g}}\right), 1.90(\mathrm{tt}, J=6.2,6.2 \mathrm{~Hz}$, $\left.2 \mathrm{H}, \mathrm{H}_{\mathrm{n}}\right), 1.59-1.53\left(\mathrm{~m}, 4 \mathrm{H}, \mathrm{H}_{\mathrm{I}}\right), 1.30\left(\mathrm{~s}, 54 \mathrm{H}, \mathrm{H}_{\mathrm{a}}, \mathrm{H}_{\mathrm{t}}\right), 1.25-0.80\left(\mathrm{~m}, 12 \mathrm{H}, \mathrm{H}_{\mathrm{J}}, \mathrm{H}_{\mathrm{K}}, \mathrm{H}_{\mathrm{L}}\right) .{ }^{13} \mathrm{C}$ NMR $\left(100 \mathrm{MHz}, 298 \mathrm{~K}, \mathrm{CDCl}_{3}\right): \delta=199.4,160.5,158.6,158.2,156.7,156.5,155.2,148.3,148.2$, $144.2(\times 2), 142.3,139.5(\times 2), 139.4,138.4,136.9,132.1(\times 2), 130.7(\times 2), 129.7,129.7,124.0$ 
$(\times 2), 123.6,121.1,119.6,114.4,114.3,112.9,112.9,72.4,72.1,67.5,66.7,64.3,63.8(\times 2), 63.0$, 36.7, $34.3(\times 2), 31.4(\times 2), 29.7,29.4,28.9,28.8,28.8,23.6$. LRES $^{+}-\mathrm{MS}: m / z=1804[\mathrm{M}+\mathrm{H}]^{+}$.

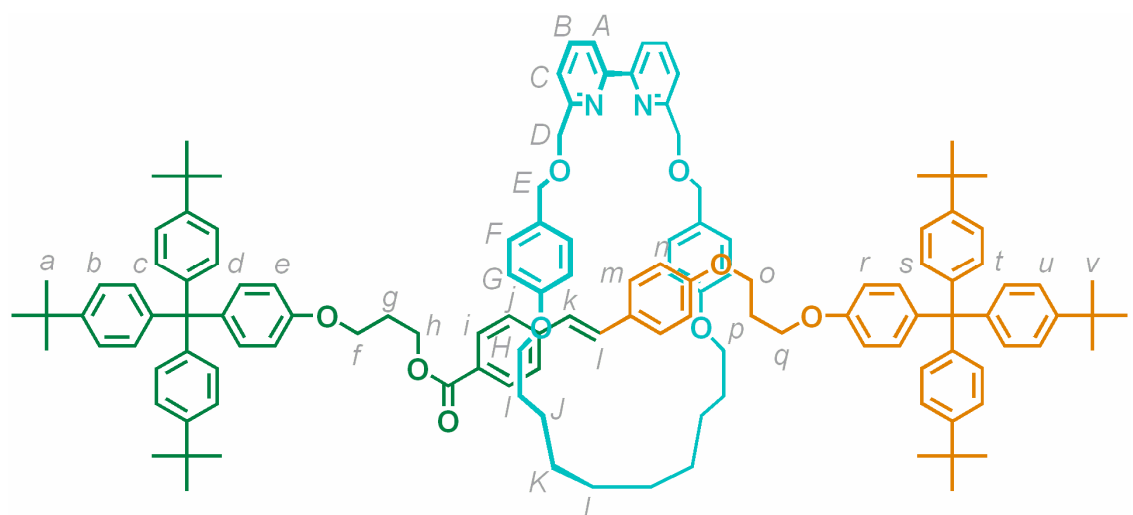

10

${ }^{1} \mathrm{H}$ NMR $\left(400 \mathrm{MHz}, \mathrm{CDCl}_{3}\right): \delta=7.93\left(\mathrm{~d}, J=7.7 \mathrm{~Hz}, 2 \mathrm{H}, \mathrm{H}_{\mathrm{A}}\right), 7.78\left(\mathrm{~d}, J=8.4 \mathrm{~Hz}, 2 \mathrm{H}, \mathrm{H}_{\mathrm{i}}\right), 7.46$ $\left(\mathrm{t}, J=7.7 \mathrm{~Hz}, 2 \mathrm{H}, \mathrm{H}_{\mathrm{B}}\right), 7.20-7.29\left(\mathrm{~m}, \mathrm{H}_{\mathrm{b}}, \mathrm{H}_{\mathrm{u}}\right.$ and $\left.\mathrm{H}_{\mathrm{C}}\right), 7.16\left(\mathrm{~d}, J=8.4 \mathrm{~Hz}, 2 \mathrm{H}, \mathrm{H}_{\mathrm{j}}\right), 7.02-7.11(\mathrm{~m}$, $\mathrm{H}_{\mathrm{c}}, \mathrm{H}_{\mathrm{d}}, \mathrm{H}_{\mathrm{s}}, \mathrm{H}_{\mathrm{t}}$ and $\left.\mathrm{H}_{\mathrm{F}}\right), 6.74\left(\mathrm{~d}, J=15.9 \mathrm{~Hz}, 1 \mathrm{H}, \mathrm{H}_{\mathrm{k}}\right), 6.51-6.68\left(\mathrm{~m}, 9 \mathrm{H}, \mathrm{H}_{\mathrm{e}}, \mathrm{H}_{\mathrm{l}}, \mathrm{H}_{\mathrm{r}}\right.$ and $\left.\mathrm{H}_{\mathrm{G}}\right), 4.55$ $\left(2 \mathrm{~s}, 8 \mathrm{H}, \mathrm{H}_{\mathrm{D}}\right.$ and $\left.\mathrm{H}_{\mathrm{E}}\right), 4.38\left(\mathrm{t}, J=6.4 \mathrm{~Hz}, 2 \mathrm{H}, \mathrm{H}_{\mathrm{h}}\right), 3.94\left(\mathrm{t}, J=6.1 \mathrm{~Hz}, 2 \mathrm{H}, \mathrm{H}_{\mathrm{f}}\right), 3.87(\mathrm{t}, J=5.8 \mathrm{~Hz}$, $2 \mathrm{H}, \mathrm{H}_{\mathrm{o}}$ or $\left.\mathrm{H}_{\mathrm{q}}\right), 3.84\left(\mathrm{t}, J=6.1 \mathrm{~Hz}, 2 \mathrm{H}, \mathrm{H}_{\mathrm{o}}\right.$ or $\left.\mathrm{H}_{\mathrm{q}}\right), 3.74\left(\mathrm{t}, J=6.4 \mathrm{~Hz}, 4 \mathrm{H}, \mathrm{H}_{\mathrm{H}}\right), 2.06-2.15(\mathrm{~m}, 2 \mathrm{H}$, $\left.\mathrm{H}_{\mathrm{g}}\right), 1.95-2.06\left(\mathrm{~m}, 2 \mathrm{H}, \mathrm{H}_{\mathrm{p}}\right), 1.51-1.60\left(\mathrm{~m}, 4 \mathrm{H}, \mathrm{H}_{\mathrm{I}}\right), 1.29\left(2 \mathrm{~s}, 54 \mathrm{H}, \mathrm{H}_{\mathrm{a}}\right.$ and $\left.\mathrm{H}_{\mathrm{v}}\right), 1.15-1.24(\mathrm{~m}, 4 \mathrm{H}$, $\left.\mathrm{H}_{\mathrm{J}}\right), 1.00-1.10\left(\mathrm{~m}, 4 \mathrm{H}, \mathrm{H}_{\mathrm{K}}\right), 0.79-0.91\left(\mathrm{~m}, 4 \mathrm{H}, \mathrm{H}_{\mathrm{L}}\right) .{ }^{13} \mathrm{C} \mathrm{NMR}\left(100 \mathrm{MHz}, \mathrm{CDCl}_{3}\right): \delta=166.2$, $158.5,158 . .3,158.1,156.5,156.4,155.2,148.2,144.2,144.2,139.5,136.9,136.8,132.3,132.1$, $132.1,131.7,130.7,130.7,130.0,129.8,129.7,129.6,129.5,128.6,127.9,127.3,124.0,124.0$ $121.1,119.6,114.4,114.3,114.0,112.8,72.4,72.4,72.1,72.1,67.5,64.1,63.9,63.0,51.7,34.3$, $34.3,31.4,31.4,29.7,29.4,28.9,28.8,28.7 .25 .8$. LRESI-MS: $m / z=1897.25[\mathrm{M}+\mathrm{H}]^{+}$ 


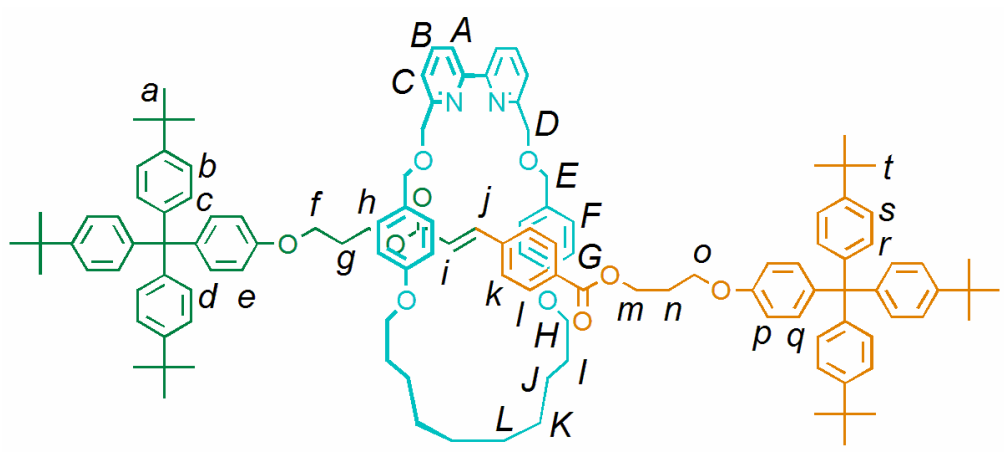

12

M.p. $94-96{ }^{\circ} \mathrm{C} .{ }^{1} \mathrm{H}$ NMR $\left(400 \mathrm{MHz}, \mathrm{CDCl}_{3}\right): \delta=7.90\left(\mathrm{~d}, J=7.7 \mathrm{~Hz}, 2 \mathrm{H}, \mathrm{H}_{\mathrm{A}}\right), 7.62(\mathrm{~d}, J=8.3$ $\left.\mathrm{Hz}, 2 \mathrm{H}, \mathrm{H}_{1}\right), 7.47\left(\mathrm{t}, J=7.7 \mathrm{~Hz}, 2 \mathrm{H}, \mathrm{H}_{\mathrm{B}}\right), 7.30\left(\mathrm{~d}, J=16.0 \mathrm{~Hz}, 1 \mathrm{H}, \mathrm{H}_{\mathrm{j}}\right), 7.25-7.28\left(\mathrm{~m}, 2 \mathrm{H}, \mathrm{H}_{\mathrm{C}}\right)$, $7.22\left(\mathrm{~d}, J=8.5 \mathrm{~Hz}, 12 \mathrm{H}, \mathrm{H}_{\mathrm{b}}\right.$ and $\left.\mathrm{H}_{\mathrm{s}}\right), 7.07\left(\mathrm{~d}, \mathrm{~J}=8.5 \mathrm{~Hz}, 16 \mathrm{H}, \mathrm{H}_{\mathrm{c}}, \mathrm{H}_{\mathrm{d}}, \mathrm{H}_{\mathrm{q}}\right.$ and $\left.\mathrm{H}_{\mathrm{r}}\right), 7.00-7.05(\mathrm{~m}$, $6 \mathrm{H}, \mathrm{H}_{\mathrm{k}}$ and $\left.\mathrm{H}_{\mathrm{F}}\right), 6.59-6.65\left(\mathrm{~m}, 8 \mathrm{H}, \mathrm{H}_{\mathrm{e}}, \mathrm{H}_{\mathrm{p}}\right.$ and $\left.\mathrm{H}_{\mathrm{G}}\right), 6.12\left(\mathrm{~d}, J=16.0 \mathrm{~Hz}, 1 \mathrm{H}, \mathrm{H}_{\mathrm{i}}\right), 4.53(2 \mathrm{~s}, 8 \mathrm{H}$, $\mathrm{H}_{\mathrm{D}}$ and $\left.\mathrm{H}_{\mathrm{E}}\right), 4.31\left(\mathrm{t}, J=6.3 \mathrm{~Hz}, 2 \mathrm{H}, \mathrm{H}_{\mathrm{m}}\right), 4.22\left(\mathrm{t}, J=6.3 \mathrm{~Hz}, 2 \mathrm{H}, \mathrm{H}_{\mathrm{h}}\right), 3.81-3.88\left(\mathrm{~m}, 4 \mathrm{H}, \mathrm{H}_{\mathrm{o}}\right.$ and $\left.\mathrm{H}_{\mathrm{f}}\right), 3.74\left(\mathrm{t}, J=6.4 \mathrm{~Hz}, 4 \mathrm{H}, \mathrm{H}_{\mathrm{H}}\right), 1.93-2.07\left(\mathrm{~m}, 4 \mathrm{H}, \mathrm{H}_{\mathrm{g}}\right.$ and $\left.\mathrm{H}_{\mathrm{n}}\right), 1.51-1.61\left(\mathrm{~m}, 4 \mathrm{H}, \mathrm{H}_{\mathrm{I}}\right), 1.29(\mathrm{~s}$, 54H, $\mathrm{H}_{\mathrm{a}}$ and $\left.\mathrm{H}_{\mathrm{t}}\right), 1.15-1.24\left(\mathrm{~m}, 4 \mathrm{H}, \mathrm{H}_{\mathrm{J}}\right), 0.78-1.15\left(\mathrm{~m}, 8 \mathrm{H}, \mathrm{H}_{\mathrm{K}}\right.$ and $\left.\mathrm{H}_{\mathrm{L}}\right) .{ }^{13} \mathrm{C} \mathrm{NMR}(100 \mathrm{MHz}$, $\left.\mathrm{CDCl}_{3}\right): \delta=166.3,165.6,158.5,158.2,156.4(\times 2), 155.1,148.2(\times 2), 144.1(\times 3), 143.1,139.6$ $(\times 2), 136.8,132.2(\times 2), 130.7(\times 2), 129.8,129.7,127.6,124.0(\times 2), 121.1,120.0,119.5,114.3$ $(\times 2), 112.8(\times 3), 72.4,72.0,67.5,63.8(\times 2), 63.0(\times 2), 61.9,61.4,34.3(\times 2), 31.4(\times 2), 29.7$, 29.4, 28.9, 28.8, 28.6, 25.8. LRFAB-MS (3-NOBA matrix): $\mathrm{m} / z=1847.9[\mathrm{M}+\mathrm{H}]^{+}$ 


\section{Characterization of the corresponding non-interlocked threads}

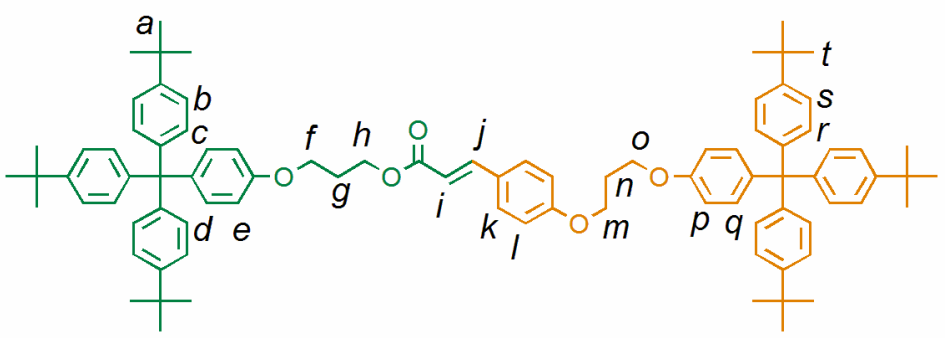

6

${ }^{1} \mathrm{H}$ NMR $\left(400 \mathrm{MHz}, \mathrm{CDCl}_{3}\right): \delta=7.63\left(\mathrm{~d}, J=15.9 \mathrm{~Hz}, 1 \mathrm{H}, \mathrm{H}_{\mathrm{j}}\right), 7.45\left(\mathrm{~d}, J=8.7 \mathrm{~Hz}, 2 \mathrm{H}, \mathrm{H}_{\mathrm{k}}\right), 7.22$ $\left(\mathrm{d}, J=8.4 \mathrm{~Hz}, 12 \mathrm{H}, \mathrm{H}_{\mathrm{b}}\right.$ and $\left.\mathrm{H}_{\mathrm{s}}\right), 7.07\left(\mathrm{~d}, J=8.6 \mathrm{~Hz}, 16 \mathrm{H}, \mathrm{H}_{\mathrm{c}}, \mathrm{H}_{\mathrm{d}}, \mathrm{H}_{\mathrm{q}}\right.$ and $\left.\mathrm{H}_{\mathrm{r}}\right), 6.89(\mathrm{~d}, J=8.7 \mathrm{~Hz}$, 2H, $\left.\mathrm{H}_{\mathrm{l}}\right), 6.77\left(\mathrm{~d}, J=8.9 \mathrm{~Hz}, 4 \mathrm{H}, \mathrm{H}_{\mathrm{e}}\right.$ and $\left.\mathrm{H}_{\mathrm{p}}\right), 6.29\left(\mathrm{~d}, J=15.9 \mathrm{~Hz}, 1 \mathrm{H}, \mathrm{H}_{\mathrm{i}}\right), 4.38(\mathrm{t}, J=6.2 \mathrm{~Hz}$, $\left.2 \mathrm{H}, \mathrm{H}_{\mathrm{h}}\right), 4.18\left(\mathrm{t}, J=6.1 \mathrm{~Hz}, 2 \mathrm{H}, \mathrm{H}_{\mathrm{m} / \mathrm{o}}\right), 4.13\left(\mathrm{t}, J=6.0 \mathrm{~Hz}, 2 \mathrm{H}, \mathrm{H}_{\mathrm{m} / \mathrm{o}}\right), 4.07\left(\mathrm{t}, J=6.1 \mathrm{~Hz}, 2 \mathrm{H}, \mathrm{H}_{\mathrm{f}}\right)$, $2.26\left(\mathrm{tt}, J=6.1,6.1 \mathrm{~Hz}, 2 \mathrm{H}, \mathrm{H}_{\mathrm{n}}\right), 2.18\left(\mathrm{tt}, J=6.2,6.0 \mathrm{~Hz}, 2 \mathrm{H}, \mathrm{H}_{\mathrm{g}}\right), 1.29\left(\mathrm{~s}, 54 \mathrm{H}, \mathrm{H}_{\mathrm{a}}\right.$ and $\left.\mathrm{H}_{\mathrm{t}}\right) ;{ }^{13} \mathrm{C}$ NMR (100 MHz, CDCl3): $\delta=167.2,160.6,156.5,156.5,148.2,148.2,144.5,144.1,144.1$, $139.7,139.6,132.2,132.2,130.6(\times 2), 129.7,127.1,124.0(\times 2), 115.4,114.8,112.9,112.8,64.6$, 64.2, 63.9, $63.0(\times 2), 61.2,34.2(\times 2), 31.3(\times 2), 29.2,28.8 ;$ LRFAB-MS $(3-N O B A): \mathrm{m} / \mathrm{z}=1253$ $[\mathrm{M}]^{+}$. HRFAB-MS (3-NOBA): $\mathrm{m} / \mathrm{z}=1252.78975$ (calc. for $\mathrm{C}_{89} \mathrm{H}_{104} \mathrm{O}_{5} 1252.78837[\mathrm{M}]^{+}$).

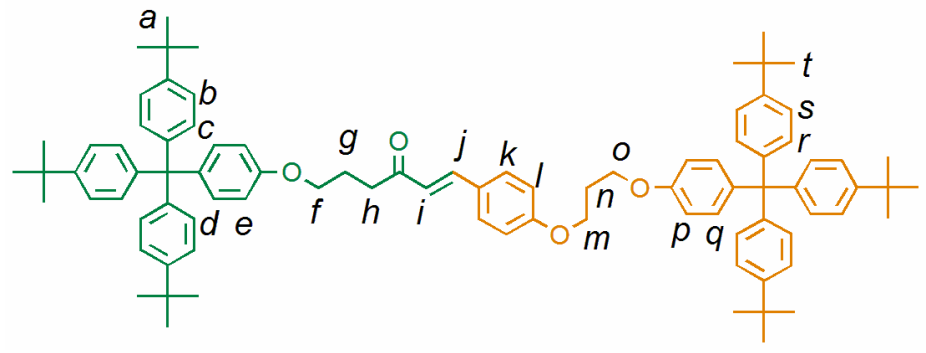

S6

${ }^{1} \mathrm{H}$ NMR $\left(400 \mathrm{MHz}, \mathrm{CDCl}_{3}\right): \delta=7.53\left(\mathrm{~d}, J=16.1 \mathrm{~Hz}, 1 \mathrm{H}, \mathrm{H}_{\mathrm{j}}\right), 7.47\left(\mathrm{~d}, J=8.8 \mathrm{~Hz}, 2 \mathrm{H}, \mathrm{H}_{\mathrm{k}}\right), 7.22$ $\left(\mathrm{d}, J=8.5 \mathrm{~Hz}, 12 \mathrm{H}, \mathrm{H}_{\mathrm{b}}\right.$ and $\left.\mathrm{H}_{\mathrm{s}}\right), 7.07\left(\mathrm{~d}, J=8.5 \mathrm{~Hz}, 16 \mathrm{H}, \mathrm{H}_{\mathrm{c}}, \mathrm{H}_{\mathrm{d}}, \mathrm{H}_{\mathrm{q}}\right.$ and $\left.\mathrm{H}_{\mathrm{r}}\right), 6.90(\mathrm{~d}, J=8.8 \mathrm{~Hz}$, $\left.2 \mathrm{H}, \mathrm{H}_{1}\right), 6.77\left(\mathrm{~d}, J=8.8 \mathrm{~Hz}, 4 \mathrm{H}, \mathrm{H}_{\mathrm{e}}\right.$ and $\left.\mathrm{H}_{\mathrm{p}}\right), 6.63\left(\mathrm{~d}, J=16.1 \mathrm{~Hz}, 1 \mathrm{H}, \mathrm{H}_{\mathrm{i}}\right), 4.18(\mathrm{t}, J=6.0 \mathrm{~Hz}$, 
$\left.2 \mathrm{H}, \mathrm{H}_{\mathrm{m} / \mathrm{o}}\right), 4.13\left(\mathrm{t}, J=6.0 \mathrm{~Hz}, 2 \mathrm{H}, \mathrm{H}_{\mathrm{m} / \mathrm{o}}\right), 4.01\left(\mathrm{t}, J=6.0 \mathrm{~Hz}, 2 \mathrm{H}, \mathrm{H}_{\mathrm{f}}\right), 2.87\left(\mathrm{t}, J=7.1 \mathrm{~Hz}, 2 \mathrm{H}, \mathrm{H}_{\mathrm{h}}\right)$, $2.26\left(\mathrm{tt}, J=6.0,6.0 \mathrm{~Hz}, 2 \mathrm{H}, \mathrm{H}_{\mathrm{n}}\right), 2.15\left(\mathrm{tt}, J=7.1,6.0 \mathrm{~Hz}, \mathrm{H}_{\mathrm{g}}\right), 1.30\left(\mathrm{~s}, 54 \mathrm{H}, \mathrm{H}_{\mathrm{a}}, \mathrm{H}_{\mathrm{t}}\right) .{ }^{13} \mathrm{C} \mathrm{NMR}$ (100 MHz, CDCl3): $\delta=199.9,161.1,156.9,156.8,148.51,148.47,144.4,144.3,142.7,140.0$ $(\mathrm{x} 2), \quad 139.8,132.5(\mathrm{x} 2), 130.9$ (x2), 130.2, 127.3, 124.3, 124.2, 114.8, 113.1 (x2), 68.0, 64.9, 64.2, $63.2(\times 2), 37.1,34.5(\times 2), 31.6(\times 2), 29.4,24.2$.

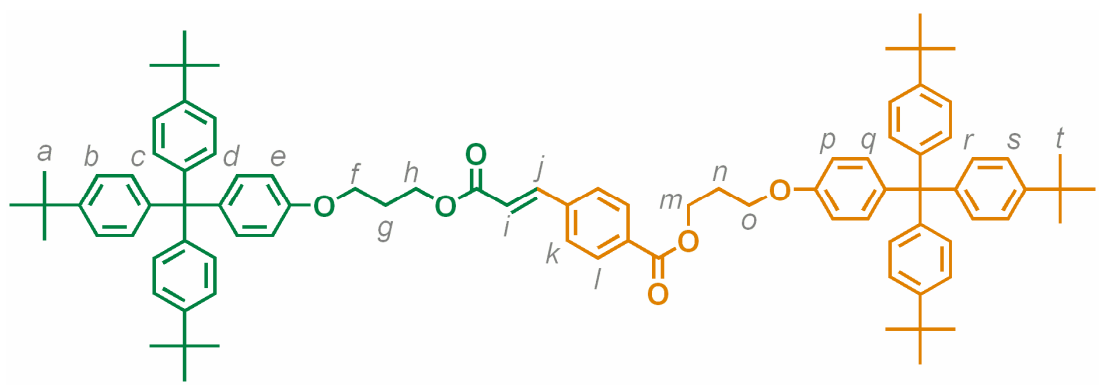

S7

Mp 152-156 ${ }^{\circ} \mathrm{C} .{ }^{1} \mathrm{H}$ NMR $\left(400 \mathrm{MHz}, \mathrm{CDCl}_{3}\right): \delta 8.03\left(\mathrm{~d}, J=8.4 \mathrm{~Hz}, 2 \mathrm{H}, \mathrm{H}_{1}\right), 7.69(\mathrm{~d}, J=16.0$ $\left.\mathrm{Hz}, 1 \mathrm{H}, \mathrm{H}_{\mathrm{j}}\right), 7.56\left(\mathrm{~d}, J=8.4 \mathrm{~Hz}, 2 \mathrm{H}, \mathrm{H}_{\mathrm{k}}\right), 8.53\left(\mathrm{~d}, J=8.5 \mathrm{~Hz}, 12 \mathrm{H}, \mathrm{H}_{\mathrm{b}}\right.$ and $\left.\mathrm{H}_{\mathrm{s}}\right), 8.56(\mathrm{~d}, J=8.5$ $\left.\mathrm{Hz}, 16 \mathrm{H}, \mathrm{H}_{\mathrm{c}}, \mathrm{H}_{\mathrm{r}}, \mathrm{H}_{\mathrm{d}}, \mathrm{H}_{\mathrm{q}}\right), 6.77\left(\mathrm{~d}, J=8.9 \mathrm{~Hz}, 4 \mathrm{H}, \mathrm{H}_{\mathrm{e}}\right.$ and $\left.\mathrm{H}_{\mathrm{p}}\right), 6.50\left(\mathrm{~d}, J=16.0 \mathrm{~Hz}, 1 \mathrm{H}, \mathrm{H}_{\mathrm{i}}\right), 4.53$ (t, $\left.J=6.3 \mathrm{~Hz}, 2 \mathrm{H}, \mathrm{H}_{\mathrm{h} / \mathrm{m}}\right), 4.42\left(\mathrm{t}, J=6.2 \mathrm{~Hz}, 2 \mathrm{H}, \mathrm{H}_{\mathrm{h} / \mathrm{m}}\right), 4.13-4.06\left(\mathrm{~m}, 4 \mathrm{H}, \mathrm{H}_{\mathrm{o}}\right.$ and $\left.\mathrm{H}_{\mathrm{f}}\right), 2.28-2.14$ (m, 4H, $\mathrm{H}_{\mathrm{g}}$ and $\left.\mathrm{H}_{\mathrm{n}}\right), 1.30\left(\mathrm{~s}, 54 \mathrm{H}, \mathrm{H}_{\mathrm{t}}\right) .{ }^{13} \mathrm{C} \mathrm{NMR}\left(100 \mathrm{MHz}, \mathrm{CDCl}_{3}\right): \delta 166.5,165.9,156.5(\mathrm{x} 2)$, 148.3 (x2), 144.1 (x2), 143.5, 139.8 (x2), 138.6, 132.3 (x2), 131.5, 130.7 (x2), 130.1, 127.9, 124.0 (x2), 120.4, $112.9(\mathrm{x} 2), 64.13,64.07,63.0,62.2,61.7$ (plus one overlaping peak in this region), 34.3(x2), $31.4(\mathrm{x} 2), 28.8(\mathrm{x} 2)$. LRFAB-MS (3-NOBA matrix): $m / z=1281[\mathrm{M}]^{+}$; HRFAB-MS (THIOG matrix): $m / z=1280.7890$ (calc. for $\mathrm{C}_{90} \mathrm{H}_{104} \mathrm{O}_{6}$ 1280.7833). 


\section{Representative Stacked ${ }^{1}$ H NMR Plots}

${ }^{1} \mathrm{H}$ NMR spectra (400 MHz, $\mathrm{CDCl}_{3}, 298 \mathrm{~K}$ ) of (a) macrocycle 1, (b) [2]rotaxane 9, (c) thread S6:

a)

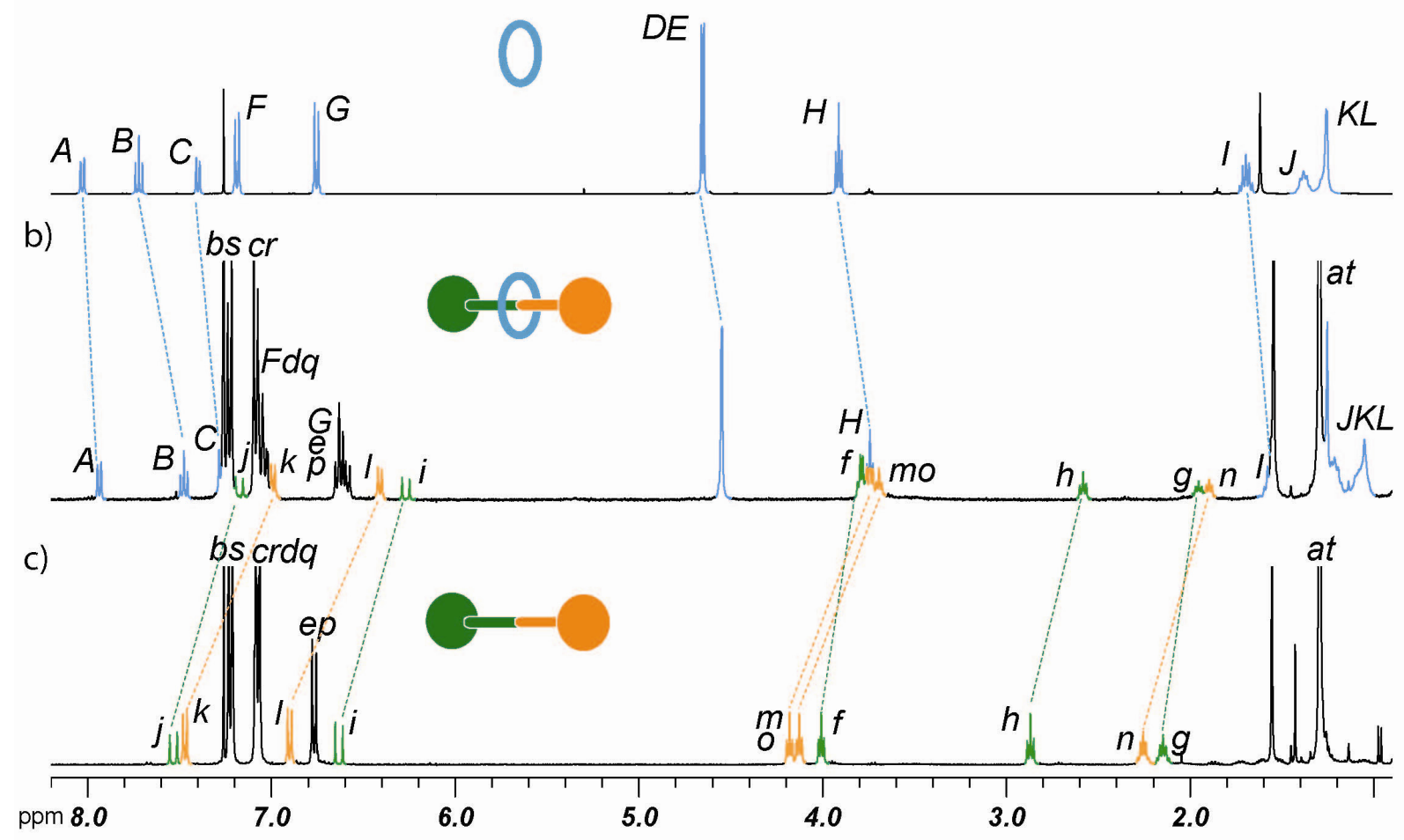


${ }^{1} \mathrm{H}$ NMR spectra (400 MHz, $\mathrm{CDCl}_{3}, 298 \mathrm{~K}$ ) of (a) macrocycle 1, (b) [2]rotaxane 12, (c) thread S7:

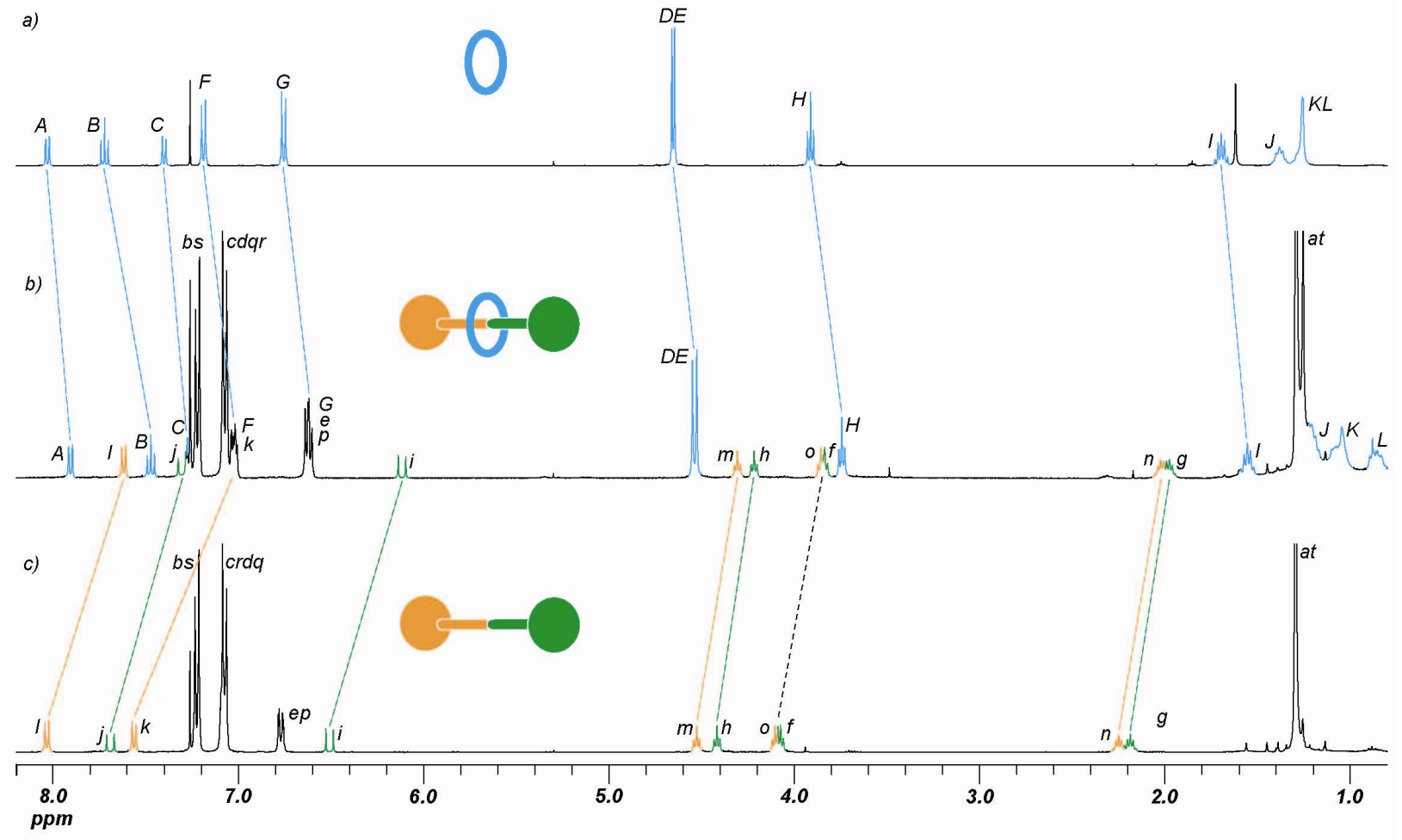




\section{Attempted Active-Template Pd(0)-Catalyzed Heck Cross-Couplings}

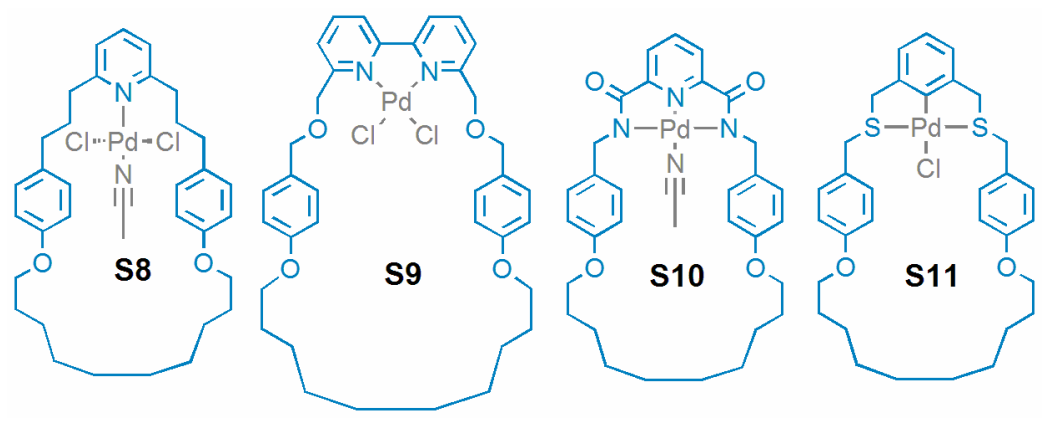

Figure 1

The Pd macrocycles S8-S11 ${ }^{1}$ shown in Figure 1 were used in the $\mathrm{Pd}(0)$ catalyzed Heck crosscouplings in an attempt to generate the corresponding [2] rotaxane (Scheme 1). Non-interlocked thread S7 was generated in good yields in all cases (80-90\%) but no desired [2] rotaxane was detected in ${ }^{1} \mathrm{H}$ NMR or ESI mass spectrometry. Similar results were obtained with Sonogashira cross-couplings.

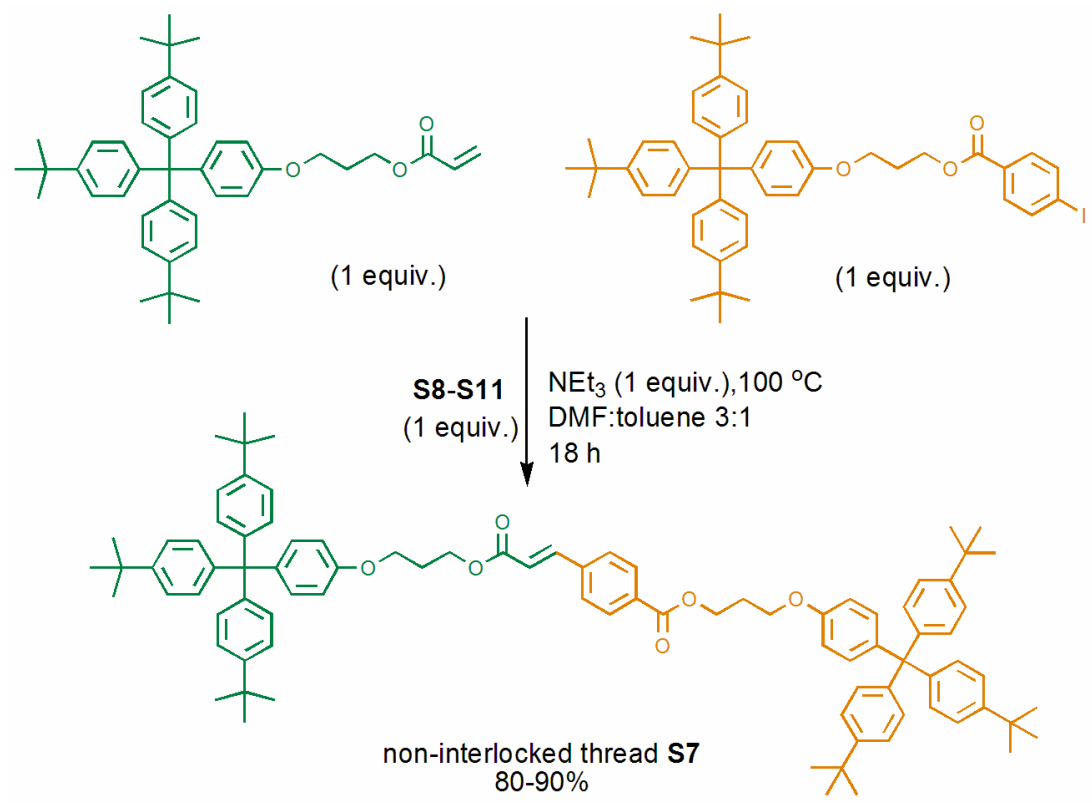

Scheme 1. $\operatorname{Pd}(0)$ catalyzed Heck cross-couplings generates only non-interlocked thread. 
Table 1 Round \#1 Optimization Studies: Preliminary Screens for the Formation of Rotaxane 5

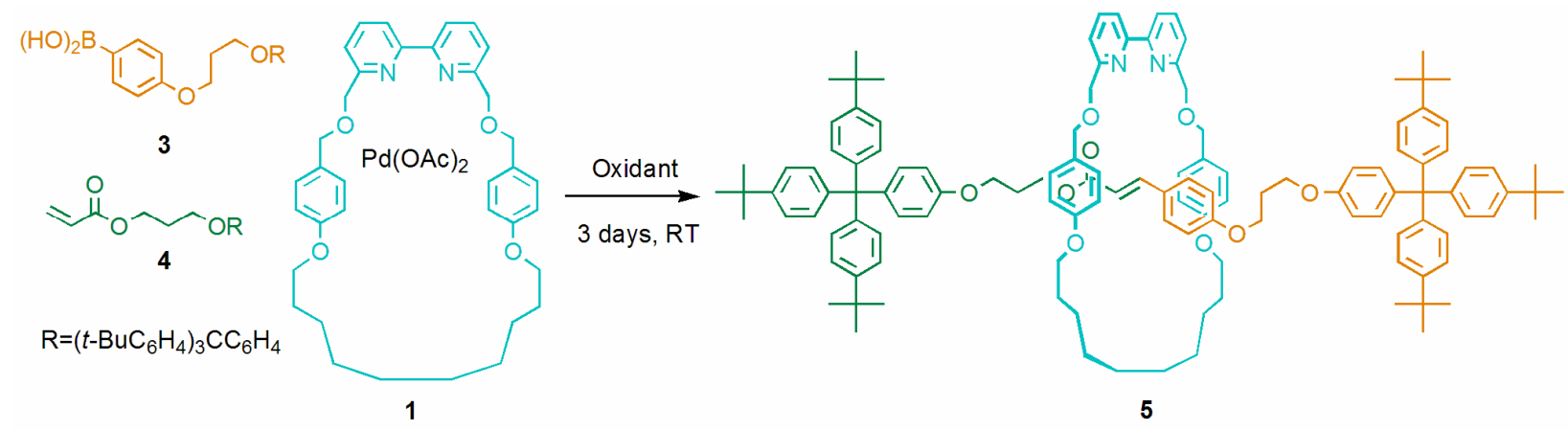

\begin{tabular}{ccccccc}
\hline Entry & $\begin{array}{c}\text { Pd(OAc) } \\
\text { (equiv.) }\end{array}$ & Oxidant & $\begin{array}{c}\text { NMM } \\
\text { (equiv.) }\end{array}$ & $\begin{array}{c}\text { Boronic acid 3 } \\
\text { (equiv.) }\end{array}$ & $\begin{array}{c}\text { Alkene } 2 \\
\text { (equiv.) }\end{array}$ & $\begin{array}{c}\text { Yield of rotaxane } \\
\mathbf{5}(\%)\end{array}$ \\
\hline $1^{\mathrm{a}}$ & 1 & air & 2 & 1 & 1 & 16 \\
$2^{\mathrm{a}}$ & 0.1 & air & 2 & 1 & 1 & 16 \\
$3^{\mathrm{a}, \mathrm{c}}$ & 0.1 & air & 2 & 1 & 1 & 11 \\
$4^{\mathrm{a}}$ & 0.1 & $\mathrm{O}_{2}$ & 2 & 1 & 1 & 16 \\
$5^{\mathrm{a}}$ & 0.1 & air & 2 & 3 & 1 & 20 \\
$6^{\mathrm{b}}$ & 0.1 & $\mathrm{O}_{2}$ & 0 & 2 & 1 & 40 \\
$7^{\mathrm{b}}$ & 0.1 & $\mathrm{O}_{2}$ & 0 & 4 & 1.5 & 32 \\
\hline
\end{tabular}

${ }^{a} \mathrm{MeCN} / \mathrm{CHCl}_{3}$ solvent system, ${ }^{\mathrm{b}} \mathrm{DMF} / \mathrm{CHCl}_{3}$ solvent system, ${ }^{\mathrm{c}}$ Reaction temperature $50{ }^{\circ} \mathrm{C}$. 
Table 2 Round \#2 Optimization Studies: Solvent and Oxidant Screen for the Formation of Rotaxane 5

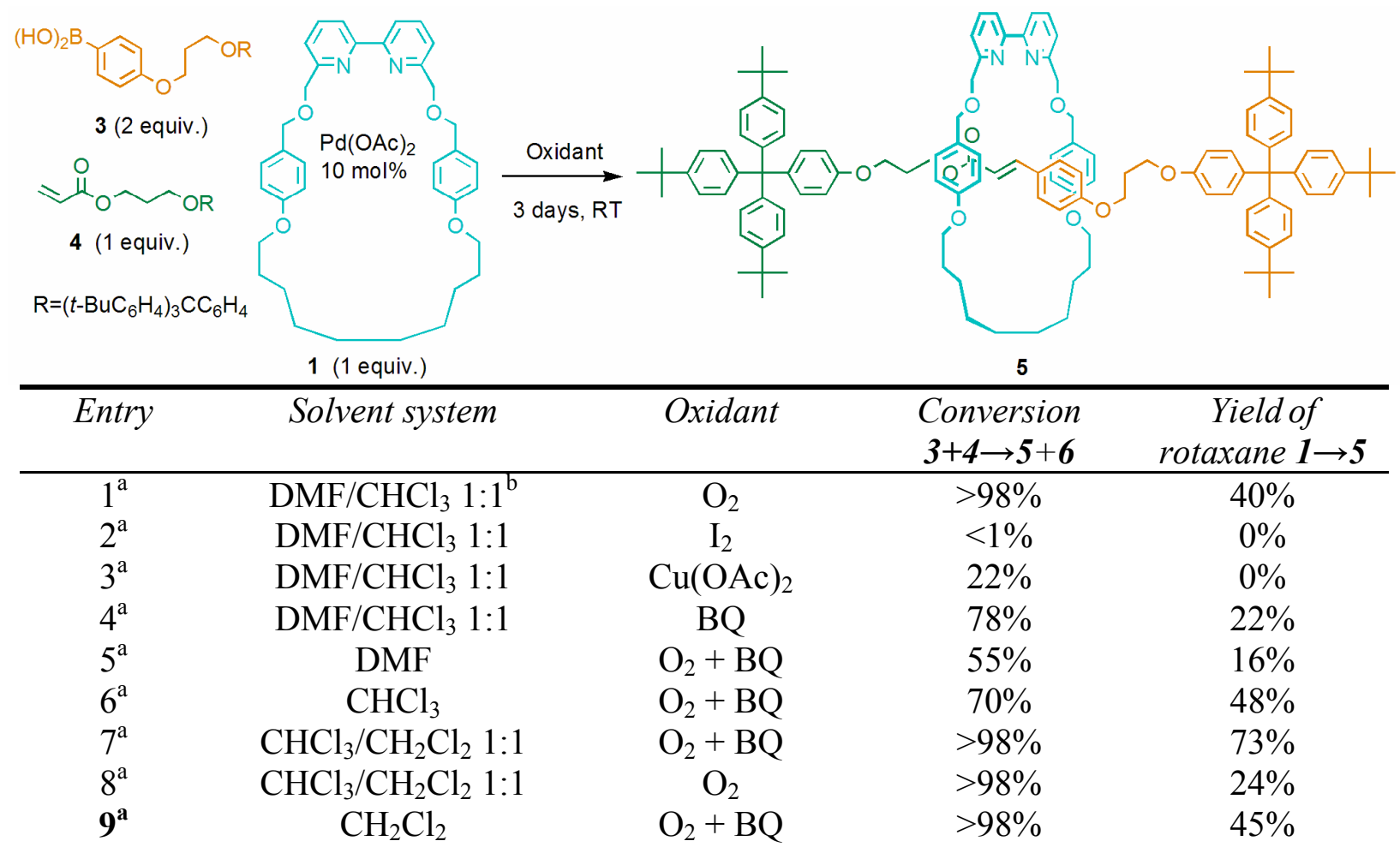

a All reactions were carried out at $16 \mathrm{mM}$ concentration with respect to 1 . bUsing $30 \mathrm{~mol} \%$ of $\mathrm{Pd}(\mathrm{OAc})_{2}$ led to $68 \%$ of conversion and $19 \%$ of [2] rotaxane $\mathbf{5}$.

Table 3 Effect of Lowering $\mathrm{Pd}(\mathrm{OAc})_{2}$ loading for the Formation of [2]Rotaxane 5

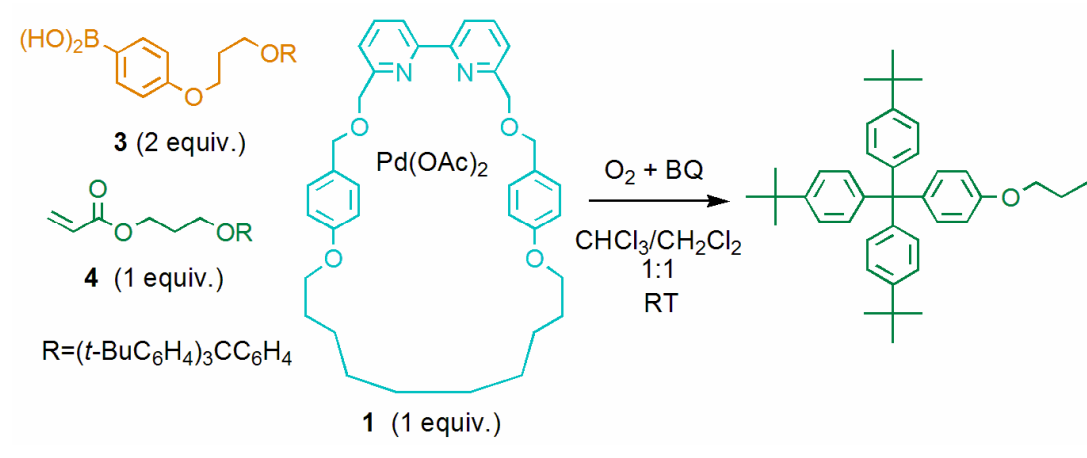

\begin{tabular}{ccccc}
\hline Entry & $P d(\mathrm{OAc})_{2}$ & Reaction Time & $\begin{array}{c}\text { Conversion } \\
\mathbf{3}+\mathbf{4} \rightarrow \mathbf{5}+\mathbf{6}\end{array}$ & $\begin{array}{c}\text { Yield of } \\
\text { rotaxane } \mathbf{1} \rightarrow \mathbf{5}\end{array}$ \\
\hline 1 & $10 \mathrm{~mol} \%$ & $72 \mathrm{~h}$ & $>98 \%$ & $73 \%$ \\
2 & $5 \mathrm{~mol} \%$ & $72 \mathrm{~h}$ & $66 \%$ & $52 \%$ \\
3 & $5 \mathrm{~mol} \%$ & $12 \mathrm{~d}$ & $>98 \%$ & $70 \%$ \\
4 & $1 \mathrm{~mol} \%$ & $72 \mathrm{~h}$ & $33 \%$ & $21 \%$ \\
5 & $1 \mathrm{~mol} \%$ & $16 \mathrm{~d}$ & $>98 \%$ & $66 \%$
\end{tabular}




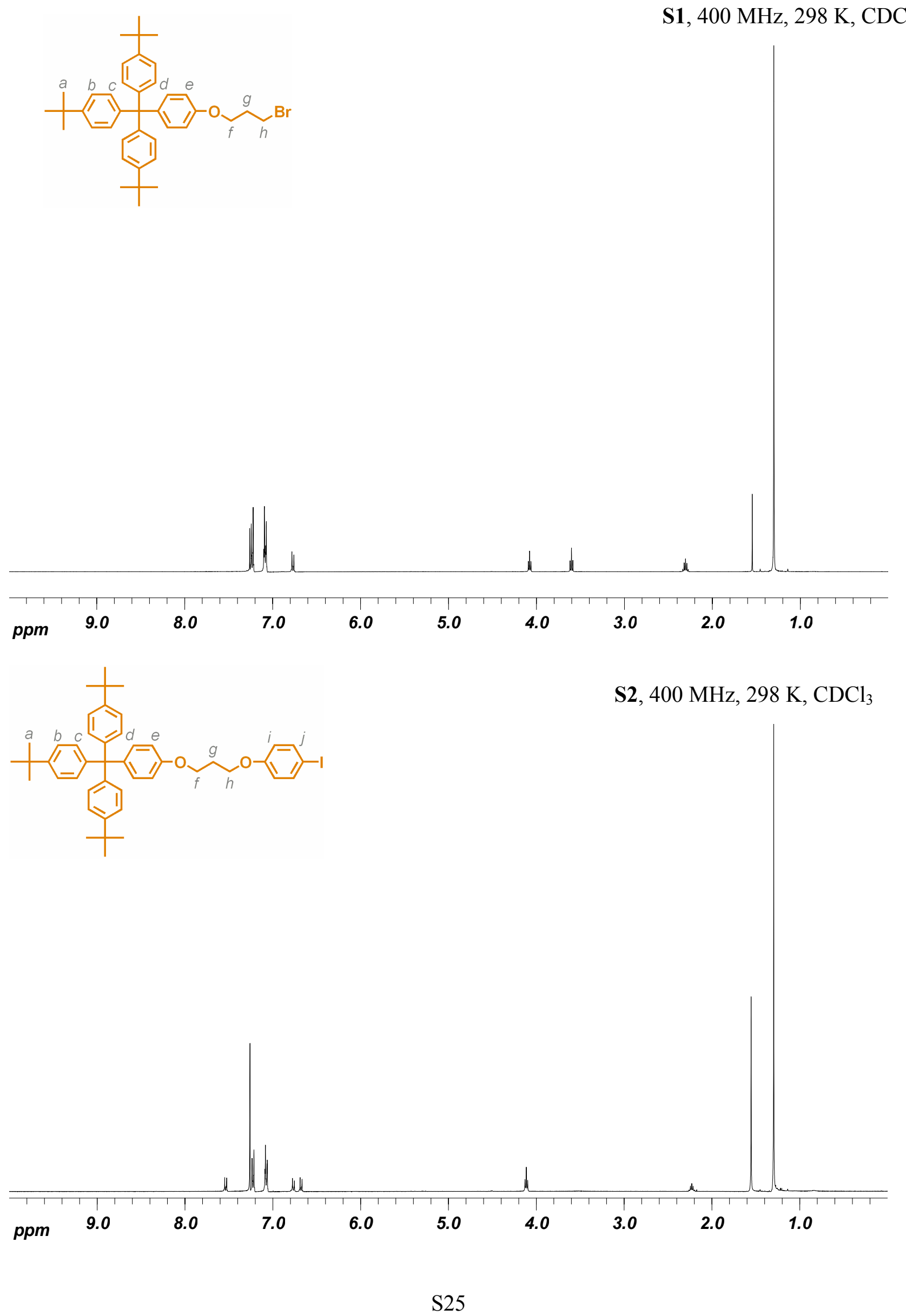


S3, $400 \mathrm{MHz}, 298 \mathrm{~K}, \mathrm{CDCl}_{3}$
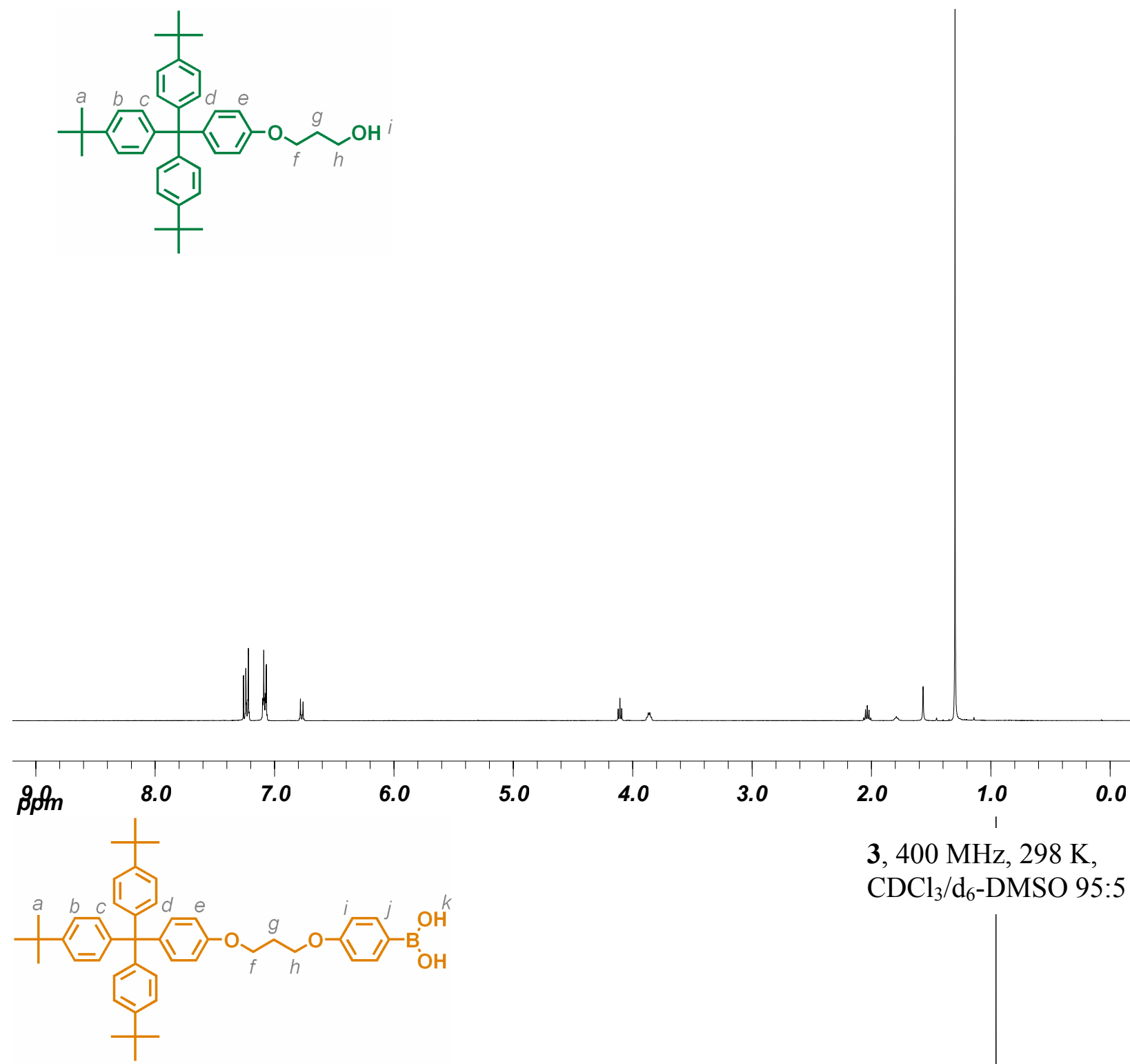

3, $400 \mathrm{MHz}, 298 \mathrm{~K}$, $\mathrm{CDCl}_{3} / \mathrm{d}_{6}$-DMSO 95:5

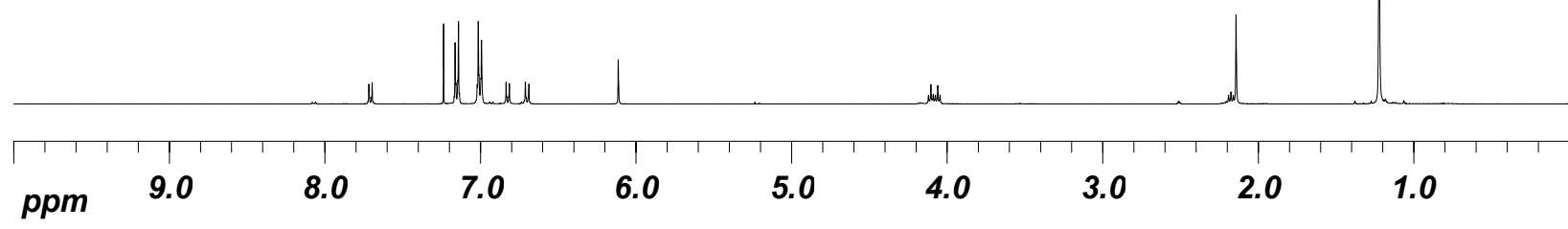




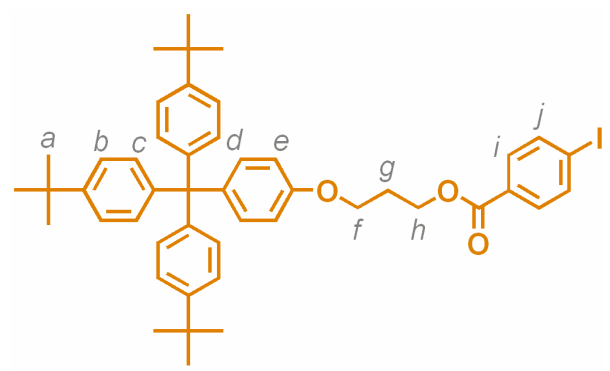

S4, $400 \mathrm{MHz}, 298 \mathrm{~K}, \mathrm{CDCl}_{3}$
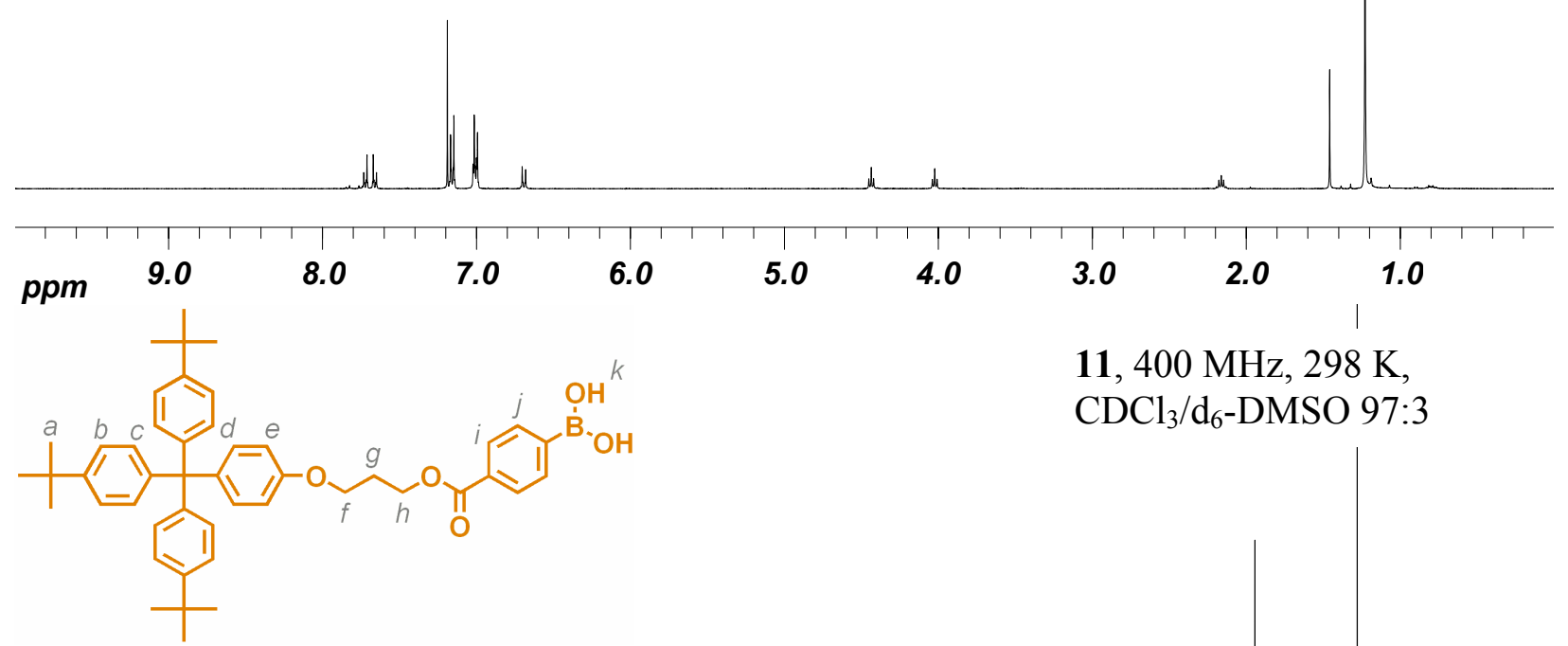

11, $400 \mathrm{MHz}, 298 \mathrm{~K}$, $\mathrm{CDCl}_{3} / \mathrm{d}_{6}$-DMSO 97:3

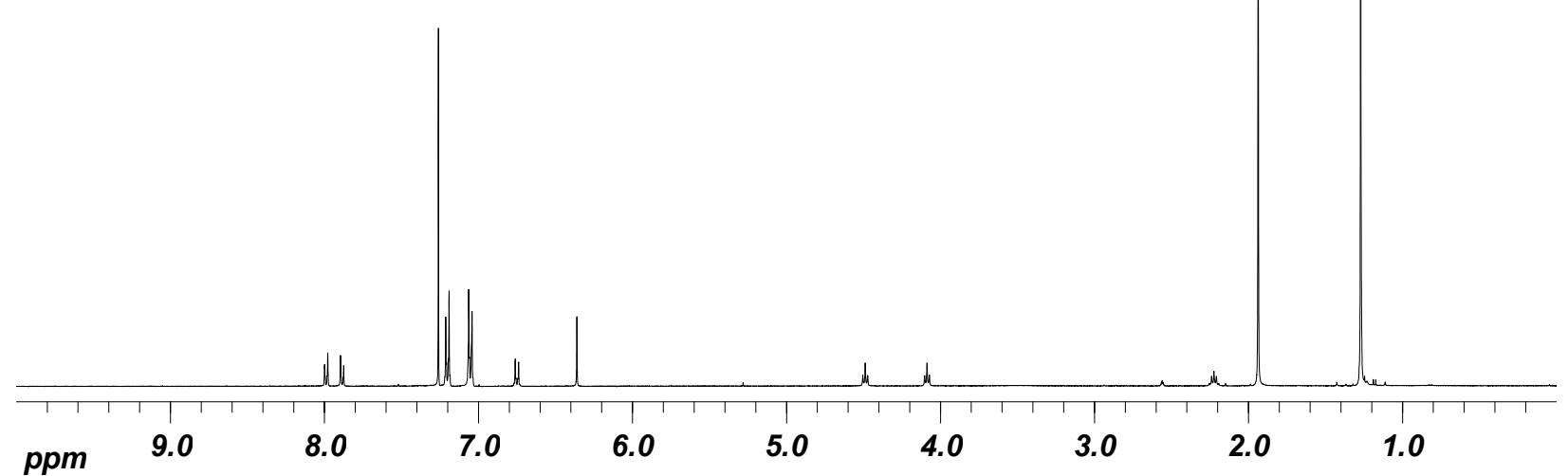




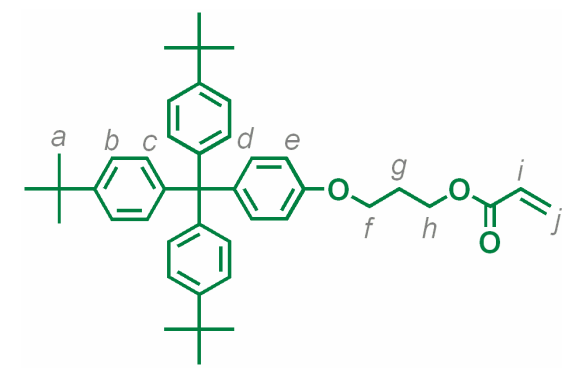

4, $400 \mathrm{MHz}, 298 \mathrm{~K}, \mathrm{CDCl}_{3}$
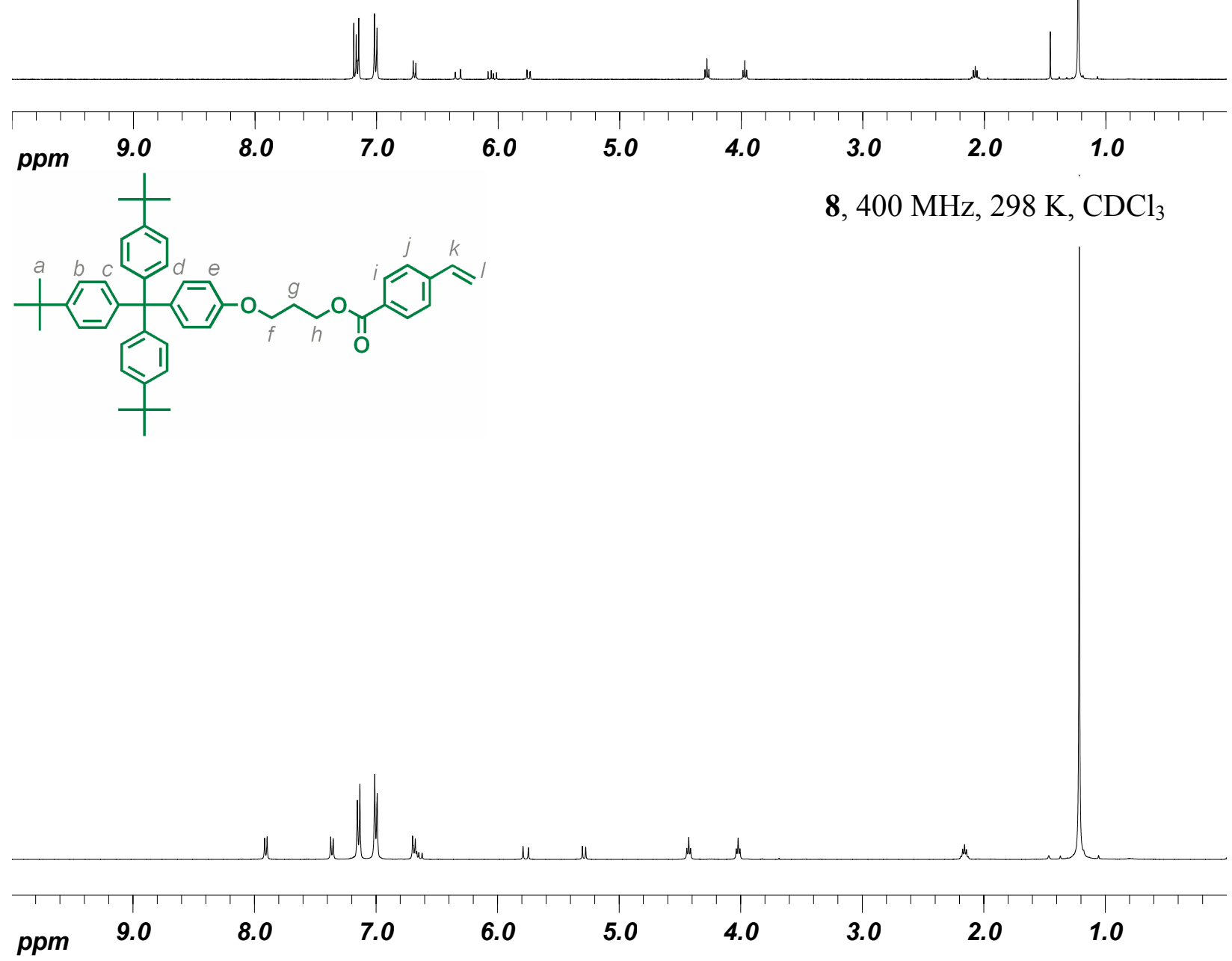


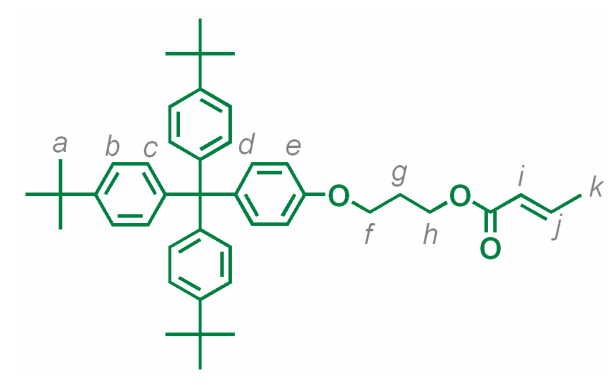

13, $400 \mathrm{MHz}, 298 \mathrm{~K}, \mathrm{CDCl}_{3}$

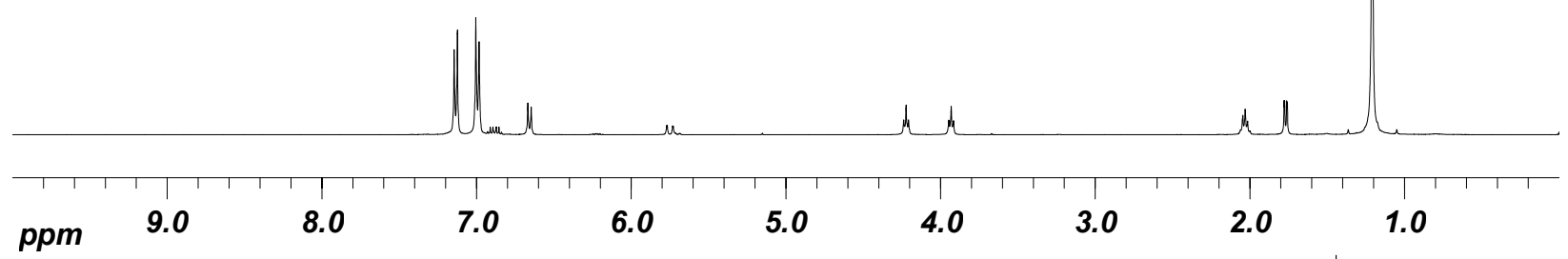

5, $400 \mathrm{MHz}, 298 \mathrm{~K}, \mathrm{CDCl}_{3}$
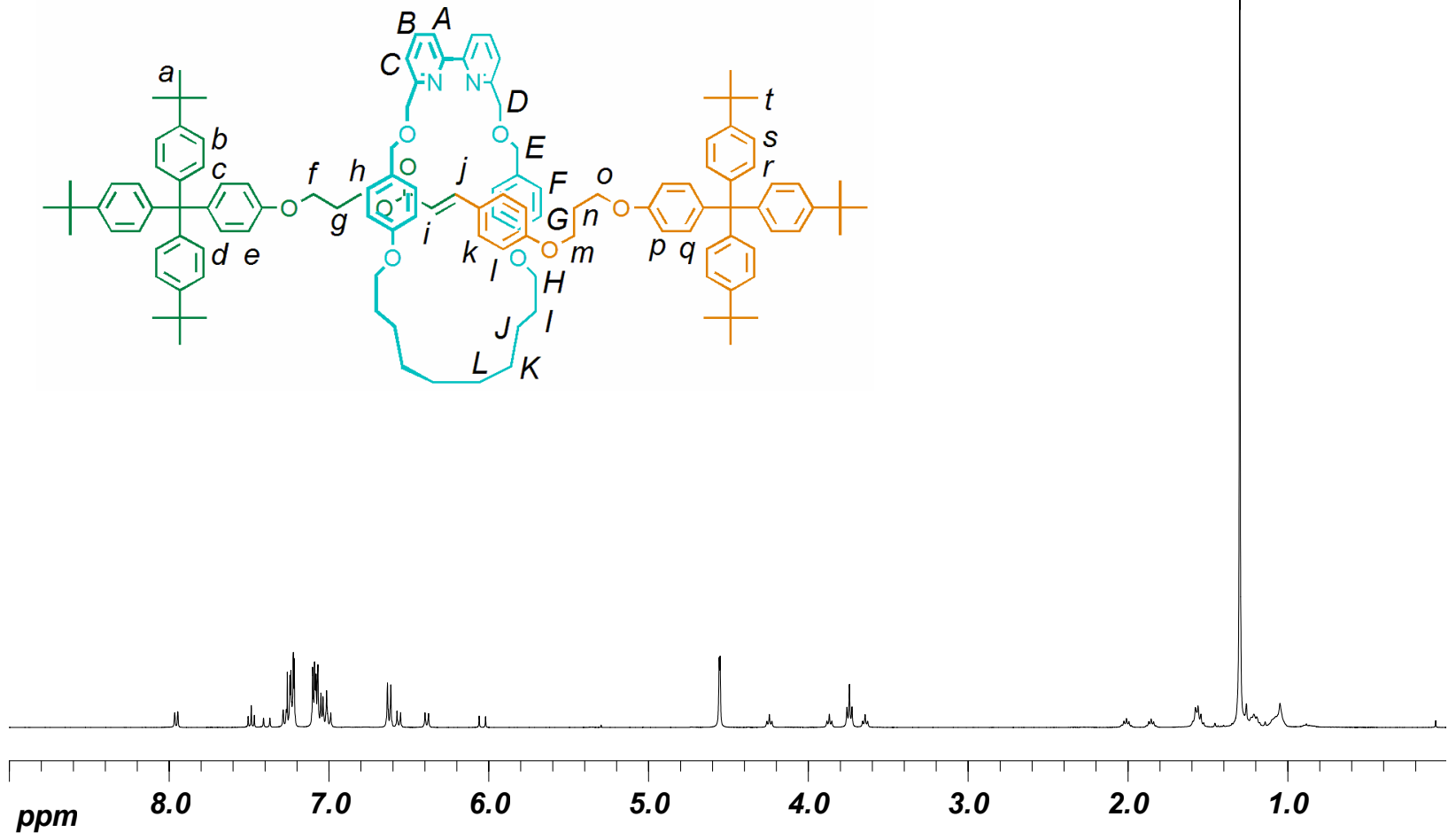
9, $400 \mathrm{MHz}, 298 \mathrm{~K}, \mathrm{CDCl}_{3}$
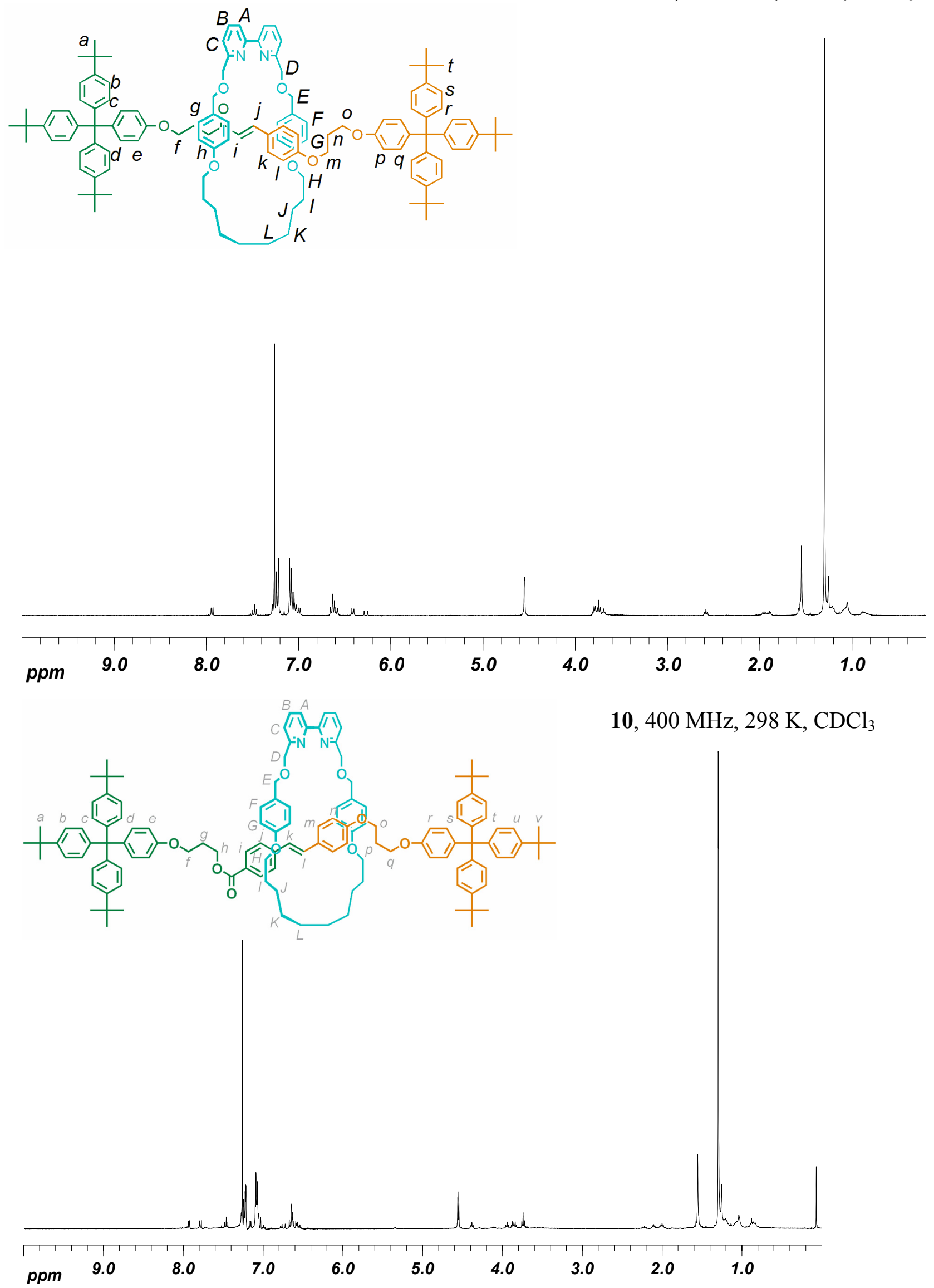
12, $400 \mathrm{MHz}, 298 \mathrm{~K}, \mathrm{CDCl}_{3}$
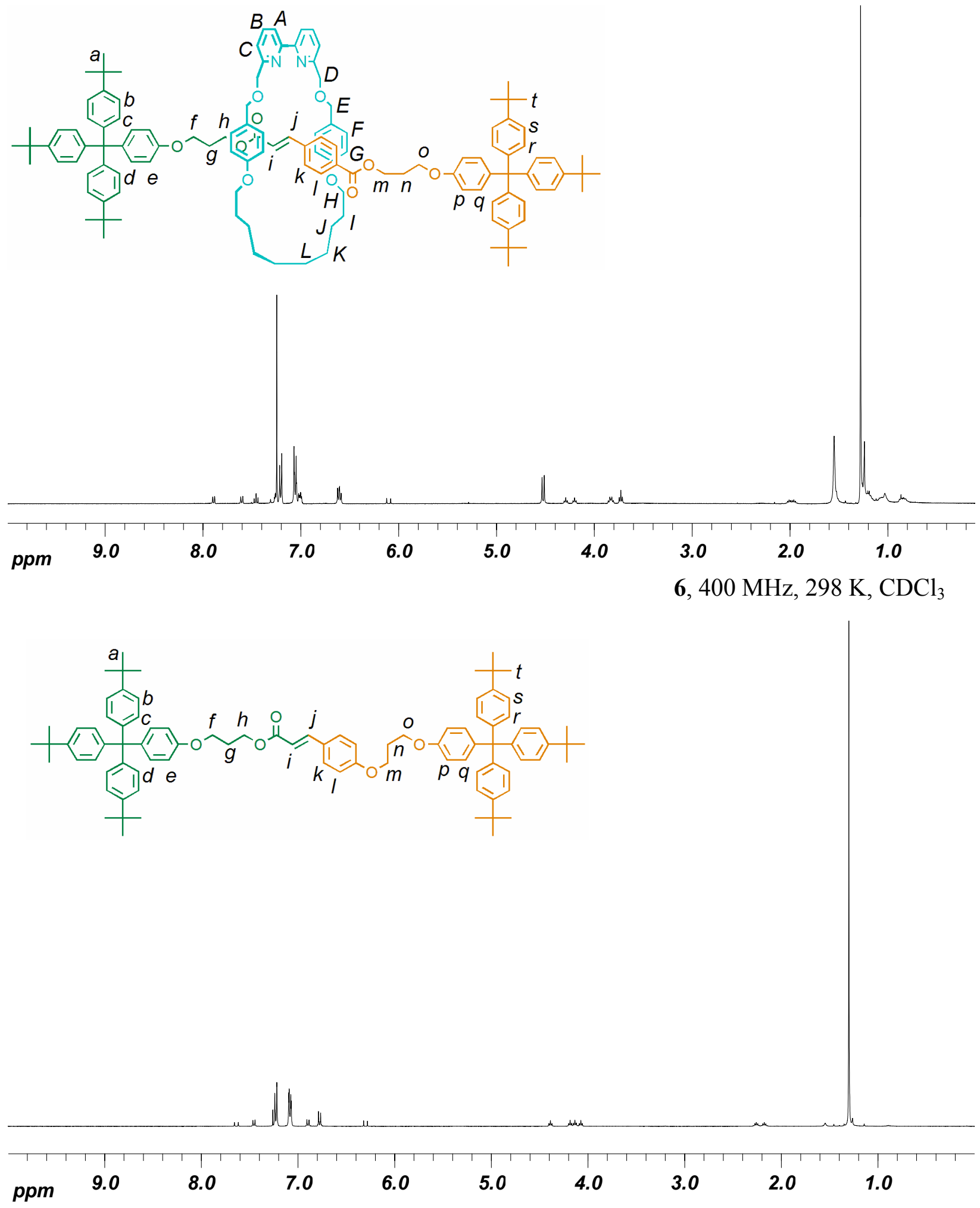


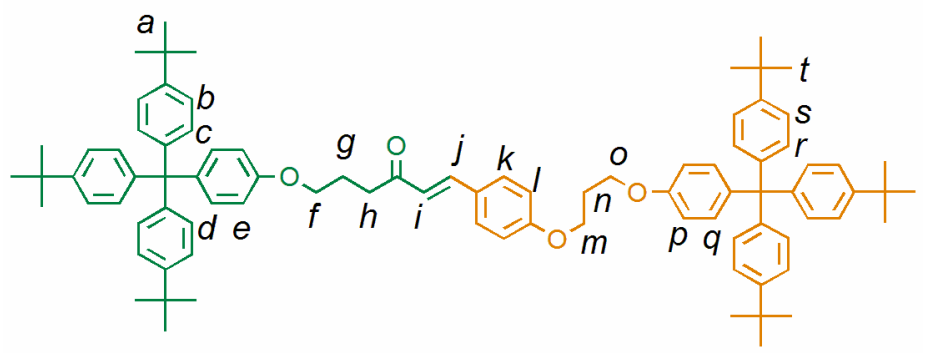

S6, $400 \mathrm{MHz}, 298 \mathrm{~K}, \mathrm{CDCl}_{3}$

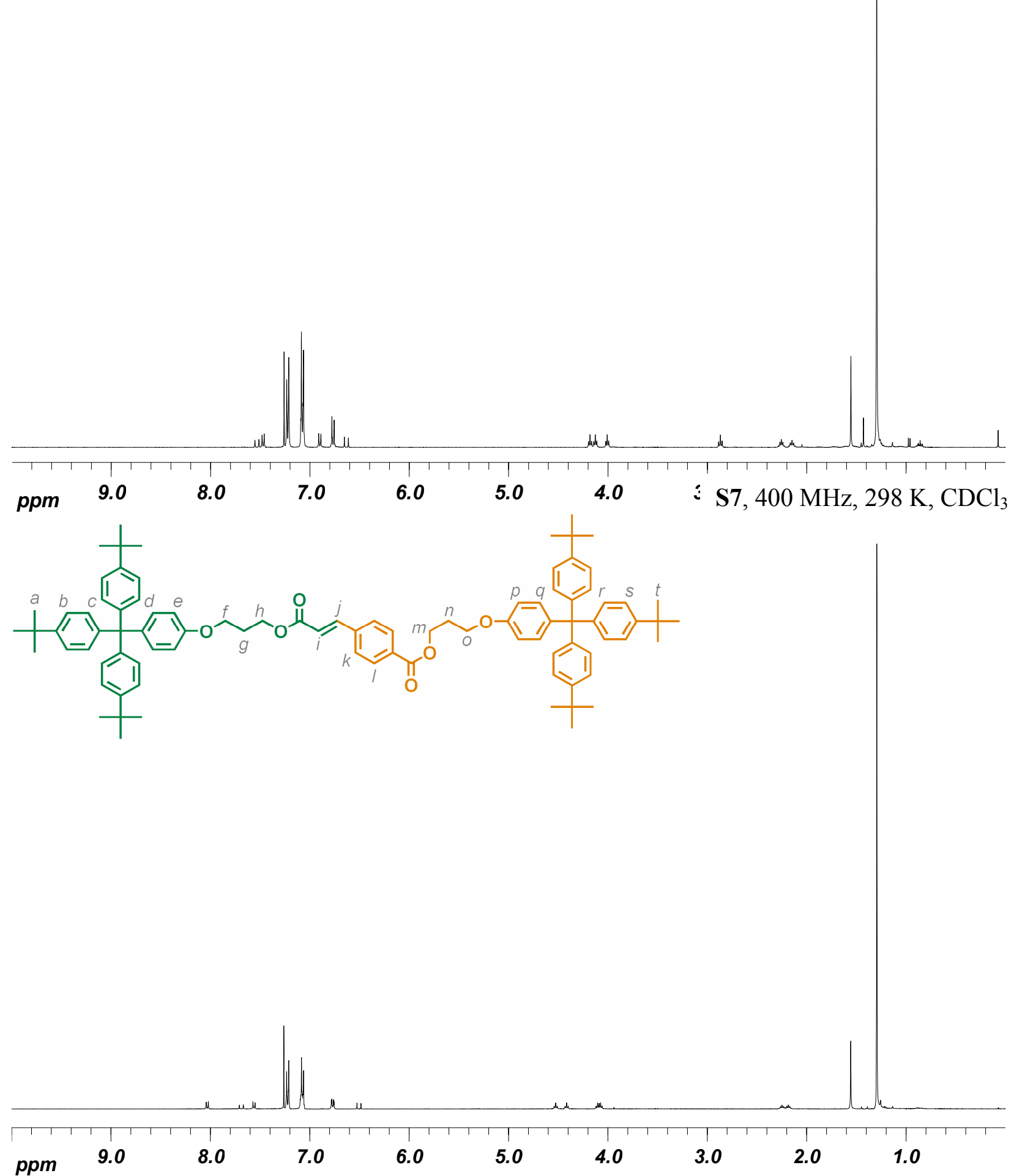




\section{References}

1) Berna, J.; Crowley, J. D.; Goldup, S. M.; Hänni, K. D.; Lee, A.-L.; Leigh, D. A.; Angew. Chem. Int. Ed. 2007, 46, 5709-5713.

2) (a) Fuller, A.-M.; Leigh, D. A.; Lusby, P. J.; Oswald, I. D. H.; Parsons, S.; Walker, D. B. Angew. Chem. Int. Ed. 2004, 43, 3914-3918. (b) Chambron, J.-C.; Sauvage, J.-P.; Mislow, K.; De Cian, A.; Fischer, J.; Chem. Eur. J. 2001, 7, 4085-4096.

3) Gibson, H. W.; Lee, S. H.; Engen, P. T.; Lecavalier, P.; Sze, J.; Shen, J.; Bheda, M. J. Org. Chem. 1993, 58, 3748-3756. 